\title{
Fluctuation theorems for capacitively coupled electronic currents
}

\author{
Gregory Bulnes Cuetara, Massimiliano Esposito, and Pierre Gaspard \\ Center for Nonlinear Phenomena and Complex Systems, Université Libre de Bruxelles, Code Postal 231, \\ Campus Plaine, B-1050 Brussels, Belgium
}

(Received 30 May 2011; revised manuscript received 2 September 2011; published 14 October 2011)

\begin{abstract}
The counting statistics of electron transport is studied theoretically in a system with two capacitively coupled parallel transport channels. Each channel is composed of a quantum dot connected by tunneling to two reservoirs. The nonequilibrium steady state of the system is controlled by two affinities or thermodynamic forces, each one determined by the two reservoirs of each channel. The status of a single-current fluctuation theorem is investigated starting from the fundamental two-current fluctuation theorem, which is a consequence of microreversibility. We show that the single-current fluctuation theorem holds in the limit of a large Coulomb repulsion between the two parallel quantum dots, as well as in the limit of a large current ratio between the parallel channels. In this latter limit, the symmetry relation of the single-current fluctuation theorem is satisfied with respect to an effective affinity that is much lower than the affinity determined by the reservoirs. This backaction effect is characterized quantitatively.
\end{abstract}

DOI: 10.1103/PhysRevB.84.165114

\section{INTRODUCTION}

Away from equilibrium, fluctuating currents flow across small open quantum systems such as quantum dots exchanging electrons with reservoirs. Advances in nonequilibrium statistical mechanics have shown that the current fluctuations obey symmetry relations following from microreversibility and known as fluctuation theorems. ${ }^{1-8}$ They have been proved in different contexts and, especially, for open quantum systems and the full counting statistics of electron transport. ${ }^{9-17}$ In this context, fluctuation theorems relate the probabilities of opposite random values of the currents to the potential differences driving the mean values of the currents. In electronic circuits, these potential differences play the role of thermodynamic forces, also called affinities. ${ }^{18,19}$ Fluctuation theorems hold in nonlinear transport regimes, particularly for the description of the Coulomb drag effect in capacitively coupled quantum dots. ${ }^{16}$

Remarkably, modern technology is able to perform the bidirectional counting of single-electron transfers in quantumdot circuits, allowing the experimental verification of the fluctuation theorem. ${ }^{20}$ In these experiments, the quantum-dot (QD) circuit is monitored by a parallel circuit made of a quantum point contact (QPC). Because of electrostatic interactions, the electronic occupancy of the quantum dots modifies the mean value of the QPC current, enabling the measurement of the QD electronic state in real time. The surprise has been that, within experimental error, the bidirectional counting of the QD current obeys the symmetry relation predicted by the fluctuation theorem but with respect to an affinity about one order of magnitude smaller than the potential difference driving the QD circuit. ${ }^{21}$ This discrepancy has revealed the importance of the interaction between the QD and QPC circuits. Indeed, the QPC current is typically $10^{7}-10^{8}$ times larger than the QD current in such experiments so that the QPC can act as a quasiclassical detector measuring the quantum state of the QD's. As a consequence, the whole system composed of the two parallel circuits is quite far from equilibrium and the shot noise in the QPC current has a significant backaction onto the small QD current. In Ref. 21, this backaction was analyzed in terms of the so-called $P(E)$ theory ${ }^{22}$ by fitting experimental data to a simple Lorentzian in order to take into account the global effect of the QPC noise onto the QD tunneling rates, an approach that has been extended in Refs. 23 and 24. These studies leave open the fundamental understanding of the backaction in terms of the microscopic Hamiltonian describing the interaction between the parallel circuits and the role of this interaction in the reduction of the effective affinity.

In the present paper, we address this issue by considering a system composed of two capacitively coupled parallel transport channels, each containing a single QD in contact with two electron reservoirs. ${ }^{16,25-28}$ The two parallel transport channels are only coupled by the electrostatic Coulomb repulsion between the electrons occupying the two QD's so that there is no electron transfer between both channels (see Fig. 1). The two currents flowing in parallel across this device are driven by two affinities defined by the potential differences on both QD's. In the analogy with the aforementioned experiments, the circuit No. 1 would play the role of the QD and the circuit No. 2 the role of the QPC detector. The fluctuations of the two currents obey a bivariate fluctuation theorem, which is the fundamental consequence of microreversibility. Under general conditions, this two-current fluctuation theorem does not imply the existence of a single-current fluctuation theorem for the main QD current monitored by the secondary circuit. Here, we show that the single-current fluctuation theorem only holds in the limit where the current in the secondary circuit is much larger than the one in the main circuit (or vice versa). However, the symmetry of the single-current fluctuation theorem does not hold with respect to the potential difference on the main circuit but to an effective affinity that depends strongly on the electrostatic interaction between both circuits. In this way, our analysis provides an understanding of these features in terms of the basic parameters of the system Hamiltonian and clearly shows that the effective affinity can vary by one order of magnitude or more due to the backaction of one circuit onto the other.

Furthermore, our analysis leads to the evaluation of the entropy production in the electronic device. The fluctuation 


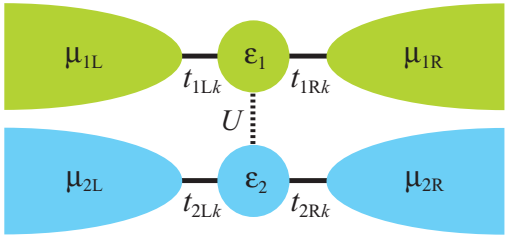

FIG. 1. (Color online) Schematic representation of two quantum dots in parallel. Each quantum dot is coupled to two reservoirs of electrons. Moreover, both quantum dots influence each other by the Coulomb electrostatic interaction.

theorem has as a consequence the non-negativity of the entropy production and is thus compatible with the second law of thermodynamics. The directionality due to the nonequilibrium driving of the device is characterized by the probability distributions of the current fluctuations, by the mean values of the currents, and also by the entropy production. The analysis based on the fluctuation theorem allows us to understand the connections between these complementary and fundamental aspects of such nonequilibrium electronic devices.

The paper is organized as follows. In Sec. II, the Hamiltonian model is presented and the master equation ruling the occupancies of the QD's is derived for QD's weakly coupled to the reservoirs within the Markovian and secular approximations. Section III is devoted to the full counting statistics of the two interacting currents, for which the fundamental fluctuation theorem is established. Moreover, the connection between the fluctuation theorem and the entropy production of the device is discussed. In Sec. IV, we first consider the limit of a large Coulomb repulsion between the QD's, in which case a single-current fluctuation theorem is obtained but without modification of the effective affinity contrary to the experimental observation. In Sec. V, the limit is then considered where the current in one circuit is much larger than the one in the other circuit. It is in this limit that the singlecurrent fluctuation theorem is obtained with an important modification of the effective affinity with respect to which the symmetry relation of the single-current fluctuation theorem holds. In Sec. VI, these effects are demonstrated numerically with the model for parameter values corresponding to typical experimental conditions. We analyze the dependence of the effective affinity on the parameters of the Hamiltonian model and, especially, on the electrostatic interaction between both circuits. Conclusions are drawn in Sec. VII.

\section{CAPACITIVELY COUPLED PARALLEL TRANSPORT CHANNELS}

\section{A. The Hamiltonian}

The vehicle of our study is the Hamiltonian model considered in Ref. 25. Each transport channel ( $\alpha=1$ or 2$)$ is composed of one quantum dot with a single energy level $\epsilon_{\alpha}$ for the electron. This level is either occupied or empty and the spin degree of freedom is ignored. Moreover, the quantum dots are capacitively coupled by electrostatic repulsion if both are occupied. This electrostatic repulsion is taken into account by an Anderson-type term with the parameter $U$. The parameter $U$ is thus the energy contribution of the Coulomb repulsion when both quantum dots are occupied by an electron. The system Hamiltonian is therefore given by

$$
H_{\mathrm{S}}=\epsilon_{1} d_{1}^{\dagger} d_{1}+\epsilon_{2} d_{2}^{\dagger} d_{2}+U d_{1}^{\dagger} d_{1} d_{2}^{\dagger} d_{2},
$$

where $d_{\alpha}$ and $d_{\alpha}^{\dagger}$ denote the annihilation and creation operators of an electron on the QD labeled by $\alpha=1,2$. This Hamiltonian is diagonalized in the four-state basis $\{|00\rangle,|10\rangle,|01\rangle,|11\rangle\}$ with the corresponding energy eigenvalues $\left\{0, \epsilon_{1}, \epsilon_{2}, \epsilon_{1}+\epsilon_{2}+\right.$ $U\}$.

Each QD is in tunneling contact with two reservoirs on its left- and right-hand sides (see Fig. 1). The system has thus four reservoirs $j=1 \mathrm{~L}, 1 \mathrm{R}, 2 \mathrm{~L}, 2 \mathrm{R}$, which are denoted as $j=\alpha i$ by the label $\alpha=1,2$ of the channel and the side $i=\mathrm{L}, \mathrm{R}$ where the reservoir stands. The Hamiltonian of all the reservoirs can be expressed in terms of the Hamiltonians $H_{j}$ of the individual reservoirs as

$$
H_{\mathrm{R}}=\sum_{j} H_{j}=\sum_{j} \sum_{k} \epsilon_{j k} c_{j k}^{\dagger} c_{j k},
$$

where $c_{j k}$ and $c_{j k}^{\dagger}$ denote the annihilation and creation operators of electrons in the corresponding states. The reservoirs are supposed to be much larger than the system itself so that the eigenvalues $\left\{\epsilon_{j k}\right\}$ of each reservoir form a very dense spectrum that is quasicontinuous and characterized by a density of states $D_{j}(\epsilon)=\sum_{k} \delta\left(\epsilon-\epsilon_{j k}\right)$. The operator giving the electron number in the reservoir $j$ is furthermore defined as $N_{j}=\sum_{k} c_{j k}^{\dagger} c_{j k}$.

The tunneling Hamiltonian establishing the interaction between the QD's and the reservoirs has the form

$$
H_{\mathrm{SR}}=\sum_{\alpha=1,2} \sum_{i=\mathrm{L}, \mathrm{R}} \sum_{k} t_{\alpha i k} d_{\alpha}^{\dagger} c_{\alpha i k}+\text { H.c., }
$$

where we have specified here the channels and the reservoirs by writing $j=\alpha i$. The effect of the electrostatic interaction on the energy barriers between the quantum dots and the reservoirs could be taken into account by including corresponding capacitances, as considered in Ref. 16. If the capacitances between the QD's and the reservoirs are included, the position of the energy levels in the QD's is in general shifted when a voltage is changed in the reservoirs. However, our primary interest here is focused essentially on the rate processes taking place in the QD's. For this purpose, we may already use the Hamiltonian model of Ref. 25 where the capacitances between the QD's and the reservoirs are absent.

Finally, the total Hamiltonian is defined as the sum

$$
H=H_{\mathrm{S}}+H_{\mathrm{R}}+H_{\mathrm{SR}} .
$$

We notice that the electron number operators of each transport channel

$$
N_{\alpha}=d_{\alpha}^{\dagger} d_{\alpha}+\sum_{i=\mathrm{L}, \mathrm{R}} \sum_{k} c_{\alpha i k}^{\dagger} c_{\alpha i k}, \quad \alpha=1,2
$$

commute separately with the total Hamiltonian

$$
\left[H, N_{1}\right]=\left[H, N_{2}\right]=0
$$

so that the electron number is conserved on each transport channel and there is no electron exchange between the channels. In contrast, the number operators of the reservoirs $N_{j}=N_{\alpha i}$ with $\alpha=1,2$ and $i=\mathrm{L}, \mathrm{R}$ do not commute with the 
total Hamiltonian unless the tunneling amplitudes are equal to zero.

\section{B. The master equation}

Initially, the reservoirs are in grand-canonical equilibrium states characterized by the chemical potentials $\mu_{j}$ with $j \in$ $\{1 \mathrm{~L}, 1 \mathrm{R}, 2 \mathrm{~L}, 2 \mathrm{R}\}$ and a uniform temperature $T$. We denote by $\beta=\left(k_{B} T\right)^{-1}$ the inverse temperature with the Boltzmann constant $k_{B}$. At the same time, the QD's are in an arbitrary statistical mixture $\rho_{\mathrm{S}}(0)$. Moreover, a measurement is performed at the initial time that determines the numbers $m_{1}$ and $m_{2}$ of electrons in the reservoirs $j=1 \mathrm{~L}$ and $j=2 \mathrm{~L}$. Consequently, the initial density matrix of the total system has the factorized form

$$
\rho_{m_{1} m_{2}}(0)=\rho_{S}(0) \prod_{j} \frac{1}{\Xi_{j}} e^{-\beta\left(H_{j}-\mu_{j} N_{j}\right)} \delta_{N_{1 \mathrm{~L}}, m_{1}} \delta_{N_{2 \mathrm{~L}}, m_{2}},
$$

where $\Xi_{j}$ denotes the partition function of the grand-canonical ensemble for the reservoir $j$. The Kronecker symbols $\delta_{N, m}$ take the unit value if $N=m$ and zero otherwise and thus they play the role of projection operators on states with a fixed number of particles. Thereafter, the density matrix of the total system evolves in time according to the Landau-von Neumann equation

$$
i \partial_{t} \rho_{m_{1} m_{2}}(t)=\left[H, \rho_{m_{1} m_{2}}(t)\right]
$$

in units where $\hbar=1$. The following normalization condition is satisfied by the initial density matrix (7) and preserved by the time evolution (8):

$$
\sum_{m_{1}, m_{2}} \operatorname{Tr} \rho_{m_{1} m_{2}}(t)=1
$$

where $\operatorname{Tr}$ denotes the trace over all the degrees of freedom of the total system.

Since we are interested in the occupancies of the QD's and the numbers of electrons transferred between the reservoirs, we focus on the probabilities $p_{\nu_{1} \nu_{2}}\left(n_{1}, n_{2}\right)$ that the QD's are in the quantum states $\left\{\left|v_{1} v_{2}\right\rangle\right\}$ with the occupancies $v_{1}=0,1$ and $\nu_{2}=0,1$, while $n_{1}$ electrons have been transferred from the reservoir $j=1 \mathrm{~L}$ to the first QD and $n_{2}$ electrons from the reservoir $j=2 \mathrm{~L}$ to the second QD between the initial time $t=0$ and the time $t$. These probabilities can be defined in terms of the density matrix of the total system according to

$$
\begin{aligned}
p_{v_{1} v_{2}}\left(n_{1}, n_{2}\right)= & \sum_{m_{1}, m_{2}} \operatorname{Tr}\left[\rho_{m_{1} m_{2}}(t)\left|v_{1} v_{2}\right\rangle\left\langle v_{1} v_{2}\right|\right. \\
& \left.\times \delta_{N_{1 \mathrm{~L}}, m_{1}-n_{1}} \delta_{N_{2 \mathrm{~L}}, m_{2}-n_{2}}\right],
\end{aligned}
$$

which results from a second measurement at time $t$ counting the numbers of transferred electrons. ${ }^{12}$

We suppose that the two quantum dots are weakly coupled to the reservoirs by small enough tunneling amplitudes $\left\{t_{j k}\right\}$ (with $j=\alpha i$ ) so that we may carry out the Born perturbative approximation on the Landau-von Neumann equation up to second order in the tunneling amplitudes. We use the secular (or rotating-wave) approximation and we take the Markovian approximation. ${ }^{12,29-31}$ As a consequence, the charging and discharging transition rates of the QD's are given, respectively, by

$$
\begin{gathered}
a_{j}=\Gamma_{j} f_{j}, \\
\bar{a}_{j}=\bar{\Gamma}_{j} \bar{f}_{j}, \\
b_{j}=\Gamma_{j}\left(1-f_{j}\right), \\
\bar{b}_{j}=\bar{\Gamma}_{j}\left(1-\bar{f}_{j}\right)
\end{gathered}
$$

in terms of the Fermi-Dirac distributions

$$
\begin{gathered}
f_{j}=\frac{1}{1+e^{\beta\left(\epsilon_{j}-\mu_{j}\right)}}, \\
\bar{f}_{j}=\frac{1}{1+e^{\beta\left(\epsilon_{j}+U-\mu_{j}\right)}},
\end{gathered}
$$

where $\epsilon_{j}=\epsilon_{\alpha}$ for $j=\alpha i$. The rate constants are given at the second order of perturbation theory by

$$
\begin{gathered}
\Gamma_{j}=2 \pi \sum_{k}\left|t_{j k}\right|^{2} \delta\left(\epsilon_{j}-\epsilon_{j k}\right)=2 \pi\left|t_{j}\left(\epsilon_{j}\right)\right|^{2} D_{j}\left(\epsilon_{j}\right), \\
\bar{\Gamma}_{j}=2 \pi \sum_{k}\left|t_{j k}\right|^{2} \delta\left(\epsilon_{j}+U-\epsilon_{j k}\right) \\
=2 \pi\left|t_{j}\left(\epsilon_{j}+U\right)\right|^{2} D_{j}\left(\epsilon_{j}+U\right),
\end{gathered}
$$

where the quantities $t_{j}(\epsilon)$ are the tunneling amplitudes as a function of energy and $D_{j}(\epsilon)$ is the density of states of the reservoir $j$.

The total system is characterized by two sets of time scales:

(1) The correlation times of the reservoirs: The correlation time of the reservoir $j$ can be estimated as $\tau_{j}^{(\mathrm{C})} \sim \Delta \epsilon_{j}^{-1}$ in terms of the width $\Delta \epsilon_{j}$ of the function giving the charging rate $a_{j}(\epsilon)=2 \pi\left|t_{j}(\epsilon)\right|^{2} D_{j}(\epsilon) f_{j}(\epsilon)$ versus the energy $\epsilon$.

(2) The relaxation times induced by the electron exchanges with the reservoirs: $\tau_{j}^{(\mathrm{R})} \sim \Gamma_{j}^{-1}$.

In consistency with the assumption of weak coupling, we suppose that the correlation times are much shorter than the relaxation times and that the secular approximation is performed by averaging the equation of motion over an intermediate time scale $\Delta t$ such that

$$
\tau_{j}^{(\mathrm{C})} \ll \Delta t \ll \tau_{j}^{(\mathrm{R})},
$$

which justifies the use of the Markovian approximation. ${ }^{29,30}$ Moreover, since the perturbative expansion is limited to second order, resonance effects are neglected. Consequently, the thermal energy should be supposed to be larger than the natural width of the QD energy levels: ${ }^{32}$

$$
\Gamma_{\alpha \mathrm{L}}+\Gamma_{\alpha \mathrm{R}} \ll k_{B} T, \quad \alpha=1,2 .
$$

Accordingly, the master equation for the probabilities

$$
\mathbf{p}\left(n_{1}, n_{2}\right)=\left(\begin{array}{l}
p_{00}\left(n_{1}, n_{2}\right) \\
p_{10}\left(n_{1}, n_{2}\right) \\
p_{01}\left(n_{1}, n_{2}\right) \\
p_{11}\left(n_{1}, n_{2}\right)
\end{array}\right)
$$

takes the form

$$
\partial_{t} \mathbf{p}\left(n_{1}, n_{2}\right)=\left(\hat{\mathrm{L}}_{1}+\hat{\mathrm{L}}_{2}\right) \cdot \mathbf{p}\left(n_{1}, n_{2}\right)
$$


with the matricial operators

$$
\hat{\mathrm{L}}_{1}=\left(\begin{array}{cccc}
-a_{1 \mathrm{~L}}-a_{1 \mathrm{R}} & b_{1 \mathrm{~L}} \hat{E}_{1}^{+}+b_{1 \mathrm{R}} & 0 & 0 \\
a_{1 \mathrm{~L}} \hat{E}_{1}^{-}+a_{1 \mathrm{R}} & -b_{1 \mathrm{~L}}-b_{1 \mathrm{R}} & 0 & 0 \\
0 & 0 & -\bar{a}_{1 \mathrm{~L}}-\bar{a}_{1 \mathrm{R}} & \bar{b}_{1 \mathrm{~L}} \hat{E}_{1}^{+}+\bar{b}_{1 \mathrm{R}} \\
0 & 0 & \bar{a}_{1 \mathrm{~L}} \hat{E}_{1}^{-}+\bar{a}_{1 \mathrm{R}} & -\bar{b}_{1 \mathrm{~L}}-\bar{b}_{1 \mathrm{R}}
\end{array}\right)
$$

and

$$
\hat{\mathrm{L}}_{2}=\left(\begin{array}{cccc}
-a_{2 \mathrm{~L}}-a_{2 \mathrm{R}} & 0 & b_{2 \mathrm{~L}} \hat{E}_{2}^{+}+b_{2 \mathrm{R}} & 0 \\
0 & -\bar{a}_{2 \mathrm{~L}}-\bar{a}_{2 \mathrm{R}} & 0 & \bar{b}_{2 \mathrm{~L}} \hat{E}_{2}^{+}+\bar{b}_{2 \mathrm{R}} \\
a_{2 \mathrm{~L}} \hat{E}_{2}^{-}+a_{2 \mathrm{R}} & 0 & -b_{2 \mathrm{~L}}-b_{2 \mathrm{R}} & 0 \\
0 & \bar{a}_{2 \mathrm{~L}} \hat{E}_{2}^{-}+\bar{a}_{2 \mathrm{R}} & 0 & -\bar{b}_{2 \mathrm{~L}}-\bar{b}_{2 \mathrm{R}}
\end{array}\right)
$$

where the step operators

$$
\hat{E}_{\alpha}^{ \pm} \equiv \exp \left( \pm \frac{\partial}{\partial n_{\alpha}}\right)
$$

increase or decrease the numbers $n_{\alpha}$ of transferred electrons

$$
\hat{E}_{\alpha}^{ \pm} \phi\left(n_{\alpha}\right)=\phi\left(n_{\alpha} \pm 1\right)
$$

when applied to any function $\phi\left(n_{\alpha}\right){ }^{33}$

We notice that the occupancy probabilities irrespective of the numbers of transferred electrons defined as

$$
P_{v_{1} v_{2}}=\sum_{n_{1}=-\infty}^{+\infty} \sum_{n_{2}=-\infty}^{+\infty} p_{v_{1} v_{2}}\left(n_{1}, n_{2}\right)
$$

obey the master equation obtained by replacing the step operators (25) by unity, $\hat{E}_{\alpha}^{ \pm}=1$, in the matricial operators (23) and (24).

\section{THE TWO-CURRENT FLUCTUATION THEOREM AND ITS CONSEQUENCES}

\section{A. The cumulant generating function and the affinities}

In order to perform the counting statistics of the electrons transferred from the left reservoirs to the quantum dots, we introduce the cumulant generating function of the currents in terms of the counting parameters $\left\{\lambda_{\alpha}\right\}$ associated with each transport channel:

$$
Q\left(\lambda_{1}, \lambda_{2}\right) \equiv \lim _{t \rightarrow \infty}-\frac{1}{t} \ln \left\langle\exp \left(-\lambda_{1} n_{1}-\lambda_{2} n_{2}\right)\right\rangle_{t},
$$

where the average

$$
\langle X\rangle \equiv \sum_{\nu_{1}, \nu_{2}, n_{1}, n_{2}} p_{v_{1} v_{2}}\left(n_{1}, n_{2}\right) X
$$

is taken with respect to the probability distribution, which is the solution of the master equation (22) at the time $t$.

We notice that the cumulant generating function (28) is given as the leading eigenvalue of the following eigenvalue problem:

$$
\mathrm{L} \cdot \mathbf{v}=-Q \mathbf{v}
$$

where

$$
\mathrm{L} \equiv e^{-\lambda \cdot \mathbf{n}}\left(\hat{\mathrm{L}}_{1}+\hat{\mathrm{L}}_{2}\right) e^{+\lambda \cdot \mathbf{n}}=\mathrm{L}_{1}+\mathrm{L}_{2}
$$

is a four-by-four matrix with real elements that depend on the counting parameters $\lambda$. Since the functions $\exp (\boldsymbol{\lambda} \cdot \mathbf{n})$ are the eigenfunctions of the step operators (25), the matrices $L_{1}$ and $\mathrm{L}_{2}$ are obtained by the following substitutions:

$$
\hat{E}_{\alpha}^{ \pm} \rightarrow e^{ \pm \lambda_{\alpha}}
$$

in Eqs. (23) and (24), as can be checked by a straightforward calculation.

The four-by-four matrix $L=L(\lambda)$ obeys the symmetry

$$
\mathrm{M}^{-1} \cdot \mathrm{L}(\boldsymbol{\lambda}) \cdot \mathrm{M}=\mathrm{L}(\mathbf{A}-\boldsymbol{\lambda})^{\mathrm{T}}
$$

with

$$
\mathbf{M}=\left(\begin{array}{cccc}
1 & 0 & 0 & 0 \\
0 & e^{-\beta\left(\epsilon_{1}-\mu_{1 \mathrm{R}}\right)} & 0 & 0 \\
0 & 0 & e^{-\beta\left(\epsilon_{2}-\mu_{2 \mathrm{R}}\right)} & 0 \\
0 & 0 & 0 & e^{-\beta\left(\epsilon_{1}+\epsilon_{2}+U-\mu_{\mathrm{RR}}-\mu_{2 \mathrm{R}}\right)}
\end{array}\right)
$$

and the affinities $\mathbf{A}=\left(A_{1}, A_{2}\right)$ defined by

$$
\begin{aligned}
& A_{1}=\ln \frac{a_{1 \mathrm{~L}} b_{1 \mathrm{R}}}{b_{1 \mathrm{~L}} a_{1 \mathrm{R}}}=\ln \frac{\bar{a}_{1 \mathrm{~L}} \bar{b}_{1 \mathrm{R}}}{\bar{b}_{1 \mathrm{~L}} \bar{a}_{1 \mathrm{R}}}=\beta\left(\mu_{1 \mathrm{~L}}-\mu_{1 \mathrm{R}}\right), \\
& A_{2}=\ln \frac{a_{2 \mathrm{~L}} b_{2 \mathrm{R}}}{b_{2 \mathrm{~L}} a_{2 \mathrm{R}}}=\ln \frac{\bar{a}_{2 \mathrm{~L}} \bar{b}_{2 \mathrm{R}}}{\bar{b}_{2 \mathrm{~L}} \bar{a}_{2 \mathrm{R}}}=\beta\left(\mu_{2 \mathrm{~L}}-\mu_{2 \mathrm{R}}\right) .
\end{aligned}
$$

We notice that these affinities can also be obtained by using Schnakenberg graph analysis.6,34 These quantities are the two independent thermodynamic forces able to drive the system away from equilibrium. The fact that there exists only two independent affinities although the system contains four reservoirs is due to the existence of the two constants of motion (6) given by the particle numbers in the two transport channels.

If the system were fully connected, only the total particle number would be a constant of motion and there would exist three independent affinities. More generally, a system composed of $r$ reservoirs and partitioned into $c$ disconnected but interacting transport channels has $c$ constant particle numbers and can be driven away from equilibrium by $r-c$ independent affinities. Here, $r=4$ and $c=2$ so that there is only $r-c=2$ independent affinities. 
As mentioned earlier, the cumulant generating function is given by the leading eigenvalue of Eq. (30), i.e., by the smallest root of the quartic characteristic polynomial:

$$
\operatorname{det}(\mathrm{L}+Q 1)=0
$$

of the four-by-four matrix (31). Therefore, the symmetry (33) implies that the cumulant generating function also obeys this symmetry. ${ }^{3,7}$ In this way, the fundamental result proves that the cumulant generating function satisfies the fluctuation theorem:

$$
Q(\lambda)=Q(\mathbf{A}-\lambda)
$$

or

$$
Q\left(\lambda_{1}, \lambda_{2}\right)=Q\left(A_{1}-\lambda_{1}, A_{2}-\lambda_{2}\right)
$$

in terms of the affinities $\mathbf{A}=\left(A_{1}, A_{2}\right)$ given by Eqs. (35) and (36).

An alternative expression of this fluctuation theorem is that the probability

$$
p\left(n_{1}, n_{2}\right)=\sum_{v_{1}, v_{2}} p_{\nu_{1} v_{2}}\left(n_{1}, n_{2}\right)
$$

for the transfer of $n_{1}$ particles in the circuit No. 1 and $n_{2}$ particles in the circuit No. 2 during the time interval $t$ obeys

$$
\frac{p\left(n_{1}, n_{2}\right)}{p\left(-n_{1},-n_{2}\right)} \simeq \exp \left(A_{1} n_{1}+A_{2} n_{2}\right) \quad \text { for } \quad t \rightarrow+\infty \text {. }
$$

Indeed, this expression implies Eq. (39) using the definition (28) of the cumulant generating function with the average (29). 6,12

In general, this two-current fluctuation theorem does not imply any single-current fluctuation theorem unless specific conditions are satisfied either by construction ${ }^{35}$ or in some particular limit, which is the case here, as shown in the following sections.

\section{B. The average currents and the response coefficients}

The average values of the particle currents are given in terms of the generating function according to

$$
J_{\alpha}=\left.\frac{\partial Q}{\partial \lambda_{\alpha}}\right|_{\lambda=0}
$$

for $\alpha=1,2$. As shown in Appendix, these currents can be expressed in terms of the probabilities (27) of the four QD states in the nonequilibrium steady state corresponding to the affinities (35) and (36) according to

$$
\begin{aligned}
& J_{1}=a_{1 \mathrm{~L}} P_{00}-b_{1 \mathrm{~L}} P_{10}+\bar{a}_{1 \mathrm{~L}} P_{01}-\bar{b}_{1 \mathrm{~L}} P_{11}, \\
& J_{2}=a_{2 \mathrm{~L}} P_{00}-b_{2 \mathrm{~L}} P_{01}+\bar{a}_{2 \mathrm{~L}} P_{10}-\bar{b}_{2 \mathrm{~L}} P_{11} .
\end{aligned}
$$

These currents are nonlinear functions of the affinities, which can be expanded in powers of the affinities in order to identify the linear- and nonlinear-response coefficients:

$$
\begin{aligned}
J_{\alpha}= & J_{\alpha}\left(A_{1}, A_{2}\right) \\
= & \sum_{\beta} L_{\alpha, \beta} A_{\beta}+\frac{1}{2} \sum_{\beta, \gamma} M_{\alpha, \beta \gamma} A_{\beta} A_{\gamma} \\
& +\frac{1}{6} \sum_{\beta, \gamma, \delta} N_{\alpha, \beta \gamma \delta} A_{\beta} A_{\gamma} A_{\delta}+\cdots .
\end{aligned}
$$

As a consequence of the fluctuation theorem (39), the linear-response coefficients $L_{\alpha, \beta}$ are given in terms of the second derivatives of the generating function with respect to the counting parameters, and they thus obey the Onsager reciprocity relations:

$$
L_{\alpha, \beta}=L_{\beta, \alpha}=-\left.\frac{1}{2} \frac{\partial^{2} Q}{\partial \lambda_{\alpha} \partial \lambda_{\beta}}\right|_{\lambda=\mathbf{0}, \mathbf{A}=\mathbf{0}} .
$$

Similar relationships have been established for the nonlinearresponse coefficients. ${ }^{36}$

The average currents as well as the linear-response coefficients can be calculated in terms of the characteristic determinant (37) of the matrix (31) as shown in Appendix. By using Eq. (A10), the Onsager coefficient turns out to be proportional to

$$
L_{1,2} \propto\left(\Gamma_{1 \mathrm{~L}} \bar{\Gamma}_{1 \mathrm{R}}-\bar{\Gamma}_{1 \mathrm{~L}} \Gamma_{1 \mathrm{R}}\right)\left(\Gamma_{2 \mathrm{~L}} \bar{\Gamma}_{2 \mathrm{R}}-\bar{\Gamma}_{2 \mathrm{~L}} \Gamma_{2 \mathrm{R}}\right) .
$$

In general, the Onsager coefficient is thus nonvanishing and there is a phenomenon of Coulomb drag according to which a current may be induced in a circuit at equilibrium if the other circuit is out of equilibrium, as shown in Ref. 16.

However, the Onsager coefficient vanishes under the condition that the rate constants of one circuit do not depend on the Coulomb repulsion parameter $U$. In this particular case, there is no Coulomb drag because

$$
J_{1}\left(0, A_{2}\right)=0 \quad \text { and } \quad J_{2}\left(A_{1}, 0\right)=0 \quad \text { if } \quad \Gamma_{j}=\bar{\Gamma}_{j} .
$$

This property is also proven in Appendix. Equation (48) implies the vanishing of the Onsager coefficient as well as the nonlinear-response coefficients allowing the coupling of one current to the affinity of the other circuit:

$$
\begin{gathered}
L_{1,2}=M_{1,22}=N_{1,222}=\cdots=0 \quad \text { and } \\
L_{2,1}=M_{2,11}=N_{2,111}=\cdots=0 \text { if } \quad \Gamma_{j}=\bar{\Gamma}_{j} .
\end{gathered}
$$

Nevertheless, these coefficients do not vanish in general.

\section{The entropy production and the energy dissipation}

A further consequence of the fluctuation theorem (39) is that the average currents (42) obey the second law of thermodynamics according to which the entropy production is always non-negative. ${ }^{6,12}$ The entropy production is the ratio between the total power dissipated in both circuits and the temperature $T$ :

$$
\frac{d_{i} S}{d t}=\frac{1}{T}\left(\Pi_{1}+\Pi_{2}\right) \geqslant 0 .
$$

The power dissipated in each circuit is defined as the product of the voltage $V_{\alpha}$ by the electric current $I_{\alpha}=e J_{\alpha}$, where $e$ is 
the electric charge of the particles: $\Pi_{\alpha}=V_{\alpha} I_{\alpha}$, with $\alpha=1,2$. This energy dissipation leads to Joule heating in the linear regime. Since the affinities are related to the voltages by

$$
A_{\alpha}=\frac{e V_{\alpha}}{k_{B} T},
$$

we have that the dissipated power in the circuit $\alpha$ is given by

$$
\Pi_{\alpha}=k_{B} T A_{\alpha} J_{\alpha}
$$

in terms of the corresponding affinity and mean current $J_{\alpha}=\lim _{t \rightarrow \infty}\left\langle n_{\alpha}\right\rangle / t$. Accordingly, the entropy production of the system characterizes the energy dissipated during the quantum measurement performed on one QD by the current flowing in the other circuit playing the role of the detector. We shall evaluate these quantities under such specific conditions in the following sections.

\section{THE LARGE COULOMB REPULSION LIMIT}

In the limit where the Coulomb repulsion between both QD's is strong, the coupling parameter $U$ takes large values so that the charging rates of a second electron on the two QD's vanish:

$$
\bar{a}_{j}=0 \quad \text { for } \quad j=1 \mathrm{~L}, 1 \mathrm{R}, 2 \mathrm{~L}, 2 \mathrm{R} \quad \text { if } \quad U=\infty .
$$

As a consequence, the probabilities (10) and (27) that the system is in the fourth state $|11\rangle$ also vanish:

$$
p_{11}\left(n_{1}, n_{2}\right)=0 \quad \text { and } \quad P_{11}=0 \quad \text { if } \quad U=\infty .
$$

In this limit, the occupancy of one QD is stopping the current in the other QD. Therefore, the secondary circuit has a nonvanishing current only when the QD No. 1 is empty and vice versa.

The cumulant generating function can thus be obtained by considering the three-by-three matrix obtained by removing the fourth row and column from the matrix (31). In this case, the characteristic determinant (37) depends on the counting parameters only in the following combinations:

$$
\begin{aligned}
& a_{1 \mathrm{R}} b_{1 \mathrm{~L}} e^{\lambda_{1}}+a_{1 \mathrm{~L}} b_{1 \mathrm{R}} e^{-\lambda_{1}}, \\
& a_{2 \mathrm{R}} b_{2 \mathrm{~L}} e^{\lambda_{2}}+a_{2 \mathrm{~L}} b_{2 \mathrm{R}} e^{-\lambda_{2}},
\end{aligned}
$$

which remain invariant under the independent substitutions $\lambda_{1} \rightarrow A_{1}-\lambda_{1}$ and/or $\lambda_{2} \rightarrow A_{2}-\lambda_{2}$ with the affinities (35) and (36). Consequently, we obtain the symmetry relations

$$
\begin{aligned}
Q\left(\lambda_{1}, \lambda_{2}\right) & =Q\left(A_{1}-\lambda_{1}, \lambda_{2}\right)=Q\left(\lambda_{1}, A_{2}-\lambda_{2}\right) \\
& =Q\left(A_{1}-\lambda_{1}, A_{2}-\lambda_{2}\right)
\end{aligned}
$$

if $U=\infty$, which implies the single-current fluctuation theorem:

$$
Q\left(\lambda_{1}, 0\right)=Q\left(A_{1}-\lambda_{1}, 0\right) \quad \text { if } \quad U=\infty
$$

but with the unmodified affinity (35). Therefore, this limit cannot explain the modification of the affinity observed in the experiments reported in Refs. 20 and 21.

\section{THE LARGE CURRENT RATIO LIMIT}

In counting statistics experiments, ${ }^{20,37}$ the secondary circuit, which is used to observe the occupancy of the QD, carries a current that is typically much larger than the current in the QD by a huge factor $10^{7}-10^{8}$. This amounts to supposing that the rate constants of circuit No. 2 are much larger than those of circuit No. 1:

$$
\Gamma_{1 \mathrm{~L}}, \Gamma_{1 \mathrm{R}}, \bar{\Gamma}_{1 \mathrm{~L}}, \bar{\Gamma}_{1 \mathrm{R}} \ll \Gamma_{2 \mathrm{~L}}, \Gamma_{2 \mathrm{R}}, \bar{\Gamma}_{2 \mathrm{~L}}, \bar{\Gamma}_{2 \mathrm{R}}
$$

Under such circumstances, the relaxation times $\tau_{1 i}^{(\mathrm{R})} \sim \Gamma_{1 i}^{-1}$ of circuit No. 1 are much longer than the relaxation times $\tau_{2 i}^{(\mathrm{R})} \sim \Gamma_{2 i}^{-1}$ of circuit No. 2 , and the monitoring of the slow circuit by the fast one is performed over a time scale $\Delta t$ such that

$$
\tau_{2 i}^{(\mathrm{R})} \ll \Delta t \ll \tau_{1 i}^{(\mathrm{R})}
$$

instead of the time scale (19).

Our aim here is to obtain the cumulant generating function for the counting statistics in the sole circuit No. 1 without measuring the current in the fast circuit No. 2, as is the case in Refs. 20 and 37. This amounts to considering the two-current generating function (28) for $\lambda_{2}=0$. Accordingly, we focus on the time evolution of the probabilities defined by

$$
p_{v_{1}}\left(n_{1}\right)=\sum_{v_{2}=0,1} \sum_{n_{2}=-\infty}^{+\infty} p_{v_{1} v_{2}}\left(n_{1}, n_{2}\right) .
$$

Since the electron transfers in circuit No. 2 are much faster than in circuit No. 1, circuit No. 2 can be supposed to be in a stationary state during the whole period when circuit No. 1 is in a given state. Such stationary states conditional to the state $v_{1}$ of the QD No. 1 are obtained by finding the zero eigenvectors of the transition matrix (24) with $\hat{E}_{2}^{ \pm}=1$. The conditional probabilities $P_{v_{2} \mid v_{1}}$ that the QD No. 2 has the occupancy $v_{2}$ provided that the QD No. 1 is in the state $v_{1}$ are given by

$$
\begin{aligned}
& P_{0 \mid 0}=\frac{b_{2}}{a_{2}+b_{2}}, \\
& P_{1 \mid 0}=\frac{a_{2}}{a_{2}+b_{2}}, \\
& P_{0 \mid 1}=\frac{\bar{b}_{2}}{\bar{a}_{2}+\bar{b}_{2}}, \\
& P_{1 \mid 1}=\frac{\bar{a}_{2}}{\bar{a}_{2}+\bar{b}_{2}},
\end{aligned}
$$

with

$$
\begin{aligned}
& a_{2}=a_{2 \mathrm{~L}}+a_{2 \mathrm{R}}, \\
& b_{2}=b_{2 \mathrm{~L}}+b_{2 \mathrm{R}}, \\
& \bar{a}_{2}=\bar{a}_{2 \mathrm{~L}}+\bar{a}_{2 \mathrm{R}}, \\
& \bar{b}_{2}=\bar{b}_{2 \mathrm{~L}}+\bar{b}_{2 \mathrm{R}} .
\end{aligned}
$$

Under the conditions (59), the probability that the system is in the state $\left|v_{1} v_{2}\right\rangle$ and that $n_{1}$ electrons have been transferred in the circuit No. 1 factorizes into the probability (61) and the probability of the occupancy $\nu_{2}$ of the QD No. 2 conditioned to the occupancy $v_{1}$ :

$$
p_{v_{1} \nu_{2}}\left(n_{1}\right)=p_{v_{1}}\left(n_{1}\right) P_{\nu_{2} \mid v_{1}} \text {. }
$$


Substituting these relations into the master equation (22) and summing over $n_{2}$ and $v_{2}$, we get the master equations for the probabilities $p_{v_{1}}\left(n_{1}\right)$ as follows:

$$
\begin{gathered}
\partial_{t} p_{0}\left(n_{1}\right)=-\left(a_{\mathrm{L}}+a_{\mathrm{R}}\right) p_{0}\left(n_{1}\right)+\left(b_{\mathrm{L}} \hat{E}_{1}^{+}+b_{\mathrm{R}}\right) p_{1}\left(n_{1}\right), \\
\partial_{t} p_{1}\left(n_{1}\right)=\left(a_{\mathrm{L}} \hat{E}_{1}^{-}+a_{\mathrm{R}}\right) p_{0}\left(n_{1}\right)-\left(b_{\mathrm{L}}+b_{\mathrm{R}}\right) p_{1}\left(n_{1}\right),
\end{gathered}
$$

where

$$
\begin{aligned}
& a_{\mathrm{L}}=a_{1 \mathrm{~L}} P_{0 \mid 0}+\bar{a}_{1 \mathrm{~L}} P_{1 \mid 0}, \\
& a_{\mathrm{R}}=a_{1 \mathrm{R}} P_{0 \mid 0}+\bar{a}_{1 \mathrm{R}} P_{1 \mid 0}, \\
& b_{\mathrm{L}}=b_{1 \mathrm{~L}} P_{0 \mid 1}+\bar{b}_{1 \mathrm{~L}} P_{1 \mid 1}, \\
& b_{\mathrm{R}}=b_{1 \mathrm{R}} P_{0 \mid 1}+\bar{b}_{1 \mathrm{R}} P_{1 \mid 1}
\end{aligned}
$$

are the charging and discharging rates of the first quantum dot averaged over the conditional stationary probabilities of the second quantum dot. The master equations (71) and (72) rule the process in the slow circuit No. 1 as monitored by the fast circuit No. 2 over the time scale (60).

Taking a solution of the form $p_{v_{1}}\left(n_{1}\right) \sim \exp \left(\lambda_{1} n_{1}-Q t\right)$ for Eqs. (71) and (72), the cumulant generating function (28) with $\lambda_{2}=0$ has thus for an approximation the leading eigenvalue of the matrix

$$
\tilde{\mathrm{L}}=\left(\begin{array}{cc}
-a_{\mathrm{L}}-a_{\mathrm{R}} & b_{\mathrm{L}} e^{+\lambda_{1}}+b_{\mathrm{R}} \\
a_{\mathrm{L}} e^{-\lambda_{1}}+a_{\mathrm{R}} & -b_{\mathrm{L}}-b_{\mathrm{R}}
\end{array}\right),
$$

which is given by

$$
Q\left(\lambda_{1}, 0\right) \simeq \frac{1}{2}\left[a_{\mathrm{L}}+a_{\mathrm{R}}+b_{\mathrm{L}}+b_{\mathrm{R}}-\sqrt{\left(a_{\mathrm{L}}+a_{\mathrm{R}}-b_{\mathrm{L}}-b_{\mathrm{R}}\right)^{2}+4\left(a_{\mathrm{L}} e^{-\lambda_{1}}+a_{\mathrm{R}}\right)\left(b_{\mathrm{L}} e^{+\lambda_{1}}+b_{\mathrm{R}}\right)}\right]
$$

in the limit (59) where the current in the second quantum dot is much larger than in the first one. In this limit, the generating function (78) obeys the single-current fluctuation theorem:

$$
Q\left(\lambda_{1}, 0\right)=Q\left(\tilde{A}_{1}-\lambda_{1}, 0\right)
$$

with the effective affinity for the first quantum dot obtained as

$$
\tilde{A}_{1} \equiv \ln \frac{a_{\mathrm{L}} b_{\mathrm{R}}}{a_{\mathrm{R}} b_{\mathrm{L}}}
$$

in terms of the averaged rates (73)-(76). This constitutes the main result of the present paper.

We notice that similar results hold in the other limit where circuit No. 1 is much faster than circuit No. 2 because both circuits have the same structure and are symmetrically coupled together through the Coulomb repulsion of parameter $U$ in Eq. (1).

The result (79) shows that the generating function of the counting statistics in the slow QD No. 1 has the symmetry of a single-current fluctuation theorem under the experimental conditions (59) but with respect to the effective affinity (80). This latter may differ by orders of magnitude with respect to the affinity (35) driving the circuit out of equilibrium. The reason for this modification is the backaction of the other circuit to which the QD is capacitively coupled. Indeed, the charging and discharging rates of the QD No. 1 are averaged over the two possible states of the QD No. 2 according to Eqs. (73)-(76) so that their effective values are modified by the backaction of circuit No. 2. This modification of the transition rates is reminiscent of the influence of environmental noises as described by the $P(E)$ theory. ${ }^{22}$

In the following section, the dependence of the effective affinity (80) on the applied voltages and other parameters is investigated numerically under specific conditions, showing the importance of the backaction effect.

\section{NUMERICAL RESULTS}

\section{A. Parameter values}

In typical counting statistics experiments, the affinities take quite large values because the voltages are large with respect to the temperature. For instance, with the voltages $V_{1}=300 \mu \mathrm{V}$, $V_{2}=800 \mu \mathrm{V}$, and the electronic temperature $T=130 \mathrm{mK}$, the affinities are given by

$$
\begin{aligned}
& A_{1}=\frac{e V_{1}}{k_{B} T}=25, \\
& A_{2}=\frac{e V_{2}}{k_{B} T}=70 .
\end{aligned}
$$

Supposing that the detector current is reduced by about $10 \%$ if the main QD is occupied, the parameter $U$ of the Coulomb repulsion between both QD's can be taken as

$$
\beta U=32.8 .
$$

Moreover, the detector current is typically about $10^{7}-10^{8}$ larger than the QD current.

The energy level of the second quantum dot is supposed to be in the middle between the reservoir chemical potentials, and the couplings to the reservoirs are chosen symmetric and independent of the energy. Under such assumptions, possible parameter values are given by

$$
\begin{gathered}
\beta \mu_{1 \mathrm{~L}}=25, \\
\beta \mu_{1 \mathrm{R}}=0, \\
\Gamma_{1 \mathrm{~L}}=\Gamma_{1 \mathrm{R}}=\bar{\Gamma}_{1 \mathrm{~L}}=\bar{\Gamma}_{1 \mathrm{R}}=1, \\
\beta \mu_{2 \mathrm{~L}}=70, \\
\beta \mu_{2 \mathrm{R}}=0, \\
\Gamma_{2 \mathrm{~L}}=\Gamma_{2 \mathrm{R}}=\bar{\Gamma}_{2 \mathrm{~L}}=\bar{\Gamma}_{2 \mathrm{R}}=10^{8}, \\
\beta \epsilon_{2}=35,
\end{gathered}
$$

while the level of the QD No. 1 has the energy $\epsilon_{1}$, which may take different values in the following numerical calculations. We suppose that the correlation times of the reservoirs are 


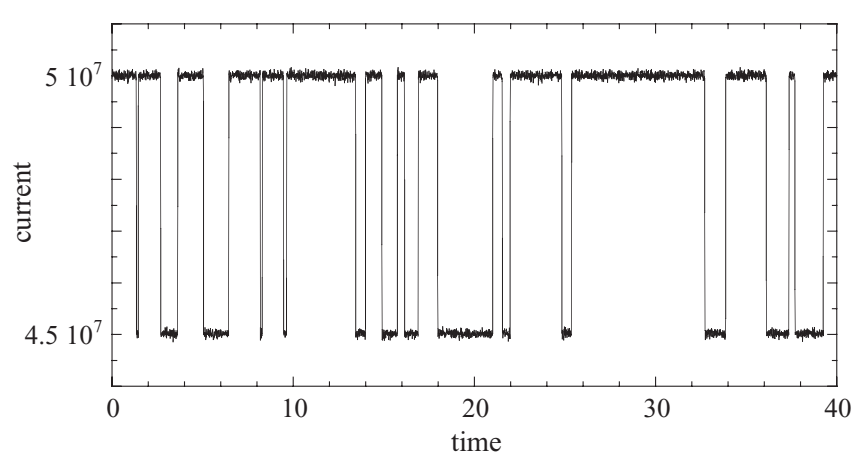

FIG. 2. Simulation with Gillespie's algorithm of the detector current in circuit No. 2 measuring the occupancy of the QD No. 1. The parameter values are given by Eqs. (83)-(90) and $\beta \epsilon_{1}=0$. The effective affinity of the circuit No. 1 is $\tilde{A}_{1}=1.17$. The mean value of the QD current is $J_{1} \simeq 0.17$ electrons per unit time. The mean value of the secondary current is $J_{2} \simeq 4.8 \times 10^{7}$ electrons per unit time. The QD is empty (occupied) when the secondary current takes the value $5 \times 10^{7}\left(4.5 \times 10^{7}\right)$.

short enough for the conditions (19) to hold in agreement with the perturbative approximation, and that the thermal energy is sufficiently large to satisfy the conditions (20). We use here the rates of the QD No. 1 in order to fix the unit of time.

There is no Coulomb drag for the conditions chosen in the present section because we have taken rate constants such that $\Gamma_{j}=\bar{\Gamma}_{j}$ in Eqs. (86) and (89). Therefore, the Onsager coefficient (47) vanishes together with higher-order coefficients according to Eqs. (48) and (49) and the Coulomb drag does not manifest itself for the conditions we consider here.

\section{B. Stochastic simulations}

The random time evolution of the system can be generated by simulating the stochastic jump process of the master equation (22) with Gillespie's algorithm. ${ }^{38,39}$ Four possible transitions may occur from each of the four states. The transition rates are given by Eqs. (11)-(14) with the FermiDirac distributions (15) and (16) and the rate constants (86) and (89).

Figure 2 depicts the current in circuit No. 2 averaged over a time interval $\Delta t=0.01$, which is shorter than the typical dwell time of the QD No. 1, as required by Eq. (60). We see that the current is reduced by about $10 \%$ when the QD No. 1 is occupied, which is in agreement with the choice for the parameter (83). The ratio between the mean values of the currents is given here by $J_{2} / J_{1}=2.8 \times 10^{8}$, while the ratio of the dissipated powers takes the value $\Pi_{2} / \Pi_{1}=\left(A_{2} J_{2}\right) /\left(A_{1} J_{1}\right)=7.9 \times 10^{8}$. Such very large ratios are required in order for the secondary current to distinguish between the two states of the QD in the primary circuit. Simulations show that the fluctuations of the secondary current would be larger for smaller values of the current ratio. Thanks to the large ratio, the instantaneous occupancy in circuit No. 1 can be monitored by the current in circuit No. 2 over the time scale (60), which is longer than the time scale of the fast circuit No. 2 but shorter than that of circuit No. 1.

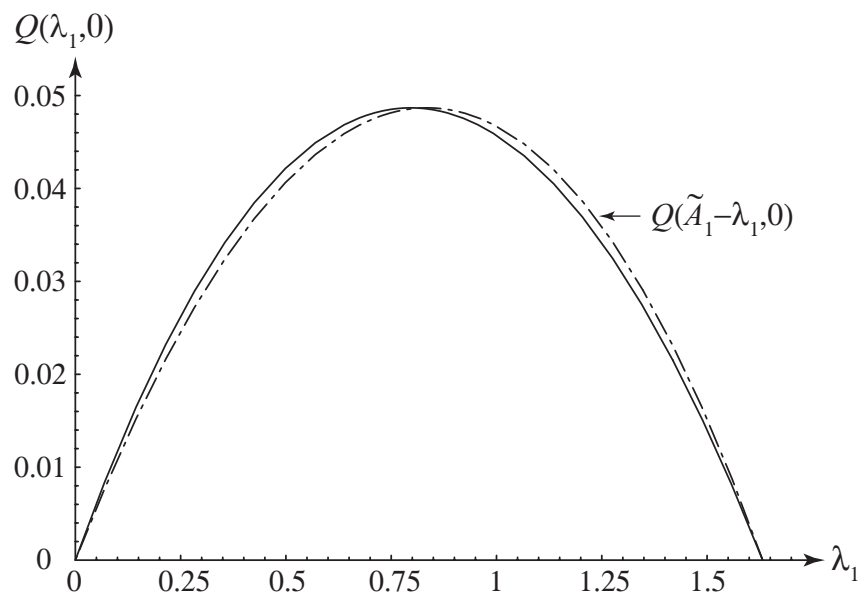

FIG. 3. The cumulant generating function vs the counting parameter $\lambda_{1}$ at $\lambda_{2}=0$ and the symmetric function with respect to the effective affinity $\tilde{A}_{1}=1.6319$ (dotted-dashed line) for the parameter values $\beta U=30, \beta \epsilon_{1}=0, \beta \epsilon_{2}=35, \beta \mu_{1 \mathrm{~L}}=25, \beta \mu_{1 \mathrm{R}}=$ $0, \beta \mu_{2 \mathrm{~L}}=70, \beta \mu_{2 \mathrm{R}}=0, \Gamma_{1 \mathrm{~L}}=\Gamma_{1 \mathrm{R}}=\bar{\Gamma}_{1 \mathrm{~L}}=\bar{\Gamma}_{1 \mathrm{R}}=1$, and $\Gamma_{2 \mathrm{~L}}=$ $\Gamma_{2 \mathrm{R}}=\bar{\Gamma}_{2 \mathrm{~L}}=\bar{\Gamma}_{2 \mathrm{R}}=1$.

\section{The cumulant generating function and its properties}

The cumulant generating function of the current in circuit No. 1 is calculated by the leading root of the characteristic polynomial (37) of the four-by-four matrix (31) with $\lambda_{2}=0$.

The lack of symmetry of the single-current generating function $Q\left(\lambda_{1}, 0\right)$ is manifest if the rate constants of both circuits are of the same order of magnitude. The generating function and its symmetric function with respect to the effective affinity is depicted in Fig. 3 for $\Gamma_{2 i} / \Gamma_{1 i}=1$ (with $i=\mathrm{L}, \mathrm{R}$ ) and $\beta U=$ 30 . Here, the effective affinity is taken as the nontrivial root of the generating function such that $Q\left(\tilde{A}_{1}, 0\right)=0$. We clearly see that the generating function is not symmetric with respect to the effective affinity $Q\left(\lambda_{1}, 0\right) \neq Q\left(\tilde{A}_{1}-\lambda_{1}, 0\right)$ so that the single-current fluctuation theorem does not hold in general although the two-current fluctuation theorem always does. Furthermore, we notice that the effective affinity $\tilde{A}_{1}=1.6319$ is much smaller than the affinity determined by the reservoirs: $A_{1}=\beta\left(\mu_{1 \mathrm{~L}}-\mu_{1 \mathrm{R}}\right)=25$.

In Fig. 4 , the single-current generating function is depicted for the smaller value of the Coulomb repulsion $\beta U=10$ and $\Gamma_{2 i} / \Gamma_{1 i}=2$. Here, the effective affinity takes a larger value, but again the asymmetry of the generating function is still manifest. The generating function now deviates from the parabolic shape seen in Fig. 3 while its maximum approaches the unity value.

Although the ratio of the rate constants is of order unity in both Figs. 3 and 4, the difference between the generating function and its symmetric function is smaller than $5 \%$ and could remain unobservable if the counting statistics were not precise enough.

Figure 5 shows the deformation of the generating function $Q\left(\lambda_{1}, 0\right)$ as the electrostatic coupling parameter $U$ varies from zero to $\beta U=20$ for $\Gamma_{2 i} / \Gamma_{1 i}=100$. In the absence of electrostatic coupling, the single-current fluctuation theorem holds in circuit No. 1 since it is decoupled from the rest of the system. In this case, the affinity takes the value $A_{1}=25$ determined by the two reservoirs of this circuit, as seen in 


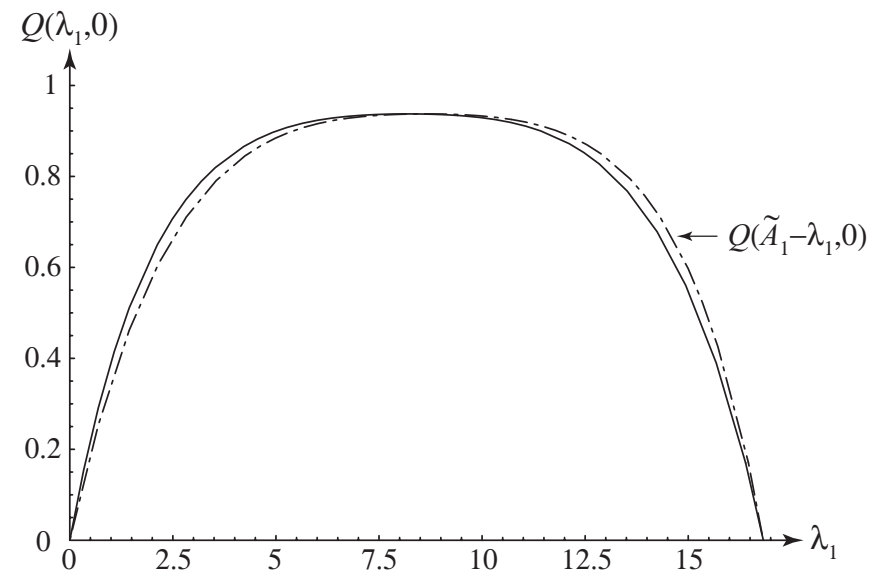

FIG. 4. The cumulant generating function vs the counting parameter $\lambda_{1}$ at $\lambda_{2}=0$ and the symmetric function with respect to the effective affinity $\tilde{A}_{1}=16.8356$ (dotted-dashed line) for the parameter values $\beta U=10, \beta \epsilon_{1}=10, \beta \epsilon_{2}=35, \beta \mu_{1 \mathrm{~L}}=25$, $\beta \mu_{1 \mathrm{R}}=0, \beta \mu_{2 \mathrm{~L}}=70, \beta \mu_{2 \mathrm{R}}=0, \Gamma_{1 \mathrm{~L}}=\Gamma_{1 \mathrm{R}}=\bar{\Gamma}_{1 \mathrm{~L}}=\bar{\Gamma}_{1 \mathrm{R}}=1$, and $\Gamma_{2 \mathrm{~L}}=\Gamma_{2 \mathrm{R}}=\bar{\Gamma}_{2 \mathrm{~L}}=\bar{\Gamma}_{2 \mathrm{R}}=2$.

Fig. 5. However, the nontrivial root $\tilde{A}_{1}$ of the generating function decreases as the Coulomb repulsion $U$ increases, showing the backaction effect of the secondary circuit due to the capacitive coupling. In the same progression, the maximum of the generating function is also reduced.

For the ratio of rate constants taken in Fig. 5, the generating function is already practically indistinguishable from its symmetric function $Q\left(\tilde{A}_{1}-\lambda_{1}, 0\right)$ so that the single-current fluctuation theorem is already effective and the considerations of Sec. V apply. In particular, the effective affinity is now approximated very well by Eq. (80).

\section{The large current ratio limit and the effective affinity}

In the limit where the ratio of rate constants tends to infinity, the generating function becomes identical with its symmetric function, as argued in Sec. V. In order to verify this prediction, we depict in Fig. 6 the difference between both functions versus the counting parameter $\lambda_{1}$. We observe in this figure that the difference is reduced by one order of magnitude each time the

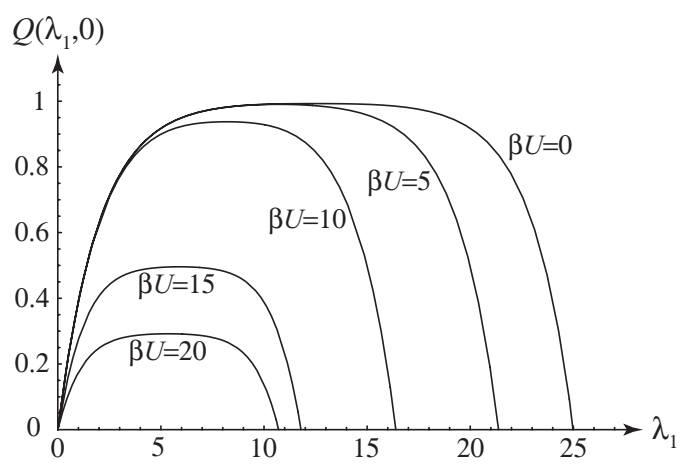

FIG. 5. The cumulant generating function vs the counting parameter $\lambda_{1}$ at $\lambda_{2}=0$ for different values of the electrostatic coupling parameter $\beta U$. The other parameters take the values $\beta \epsilon_{1}=10$, $\beta \epsilon_{2}=35, \beta \mu_{1 \mathrm{~L}}=25, \beta \mu_{1 \mathrm{R}}=0, \beta \mu_{2 \mathrm{~L}}=70, \beta \mu_{2 \mathrm{R}}=0, \Gamma_{1 \mathrm{~L}}=$ $\Gamma_{1 \mathrm{R}}=\bar{\Gamma}_{1 \mathrm{~L}}=\bar{\Gamma}_{1 \mathrm{R}}=1$, and $\Gamma_{2 \mathrm{~L}}=\Gamma_{2 \mathrm{R}}=\bar{\Gamma}_{2 \mathrm{~L}}=\bar{\Gamma}_{2 \mathrm{R}}=100$.

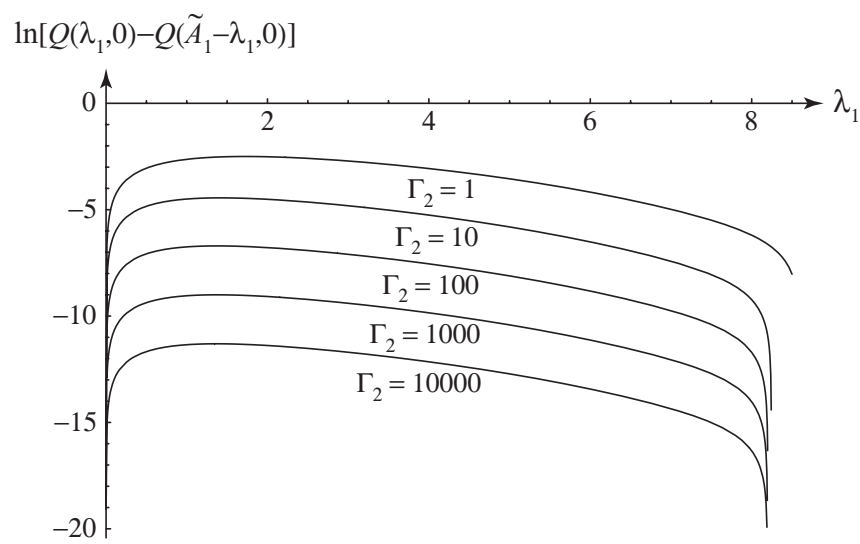

FIG. 6. The difference between the cumulant generating function and its symmetric with respect to the effective affinity $\tilde{A}_{1}$ vs the counting parameter $\lambda_{1}$ at $\lambda_{2}=0$ for the parameter values $\beta U=10$, $\beta \epsilon_{1}=10, \beta \epsilon_{2}=35, \beta \mu_{1 \mathrm{~L}}=25, \beta \mu_{1 \mathrm{R}}=0, \beta \mu_{2 \mathrm{~L}}=70, \beta \mu_{2 \mathrm{R}}=0$, $\Gamma_{1 \mathrm{~L}}=\Gamma_{1 \mathrm{R}}=\bar{\Gamma}_{1 \mathrm{~L}}=\bar{\Gamma}_{1 \mathrm{R}}=1$, and $\Gamma_{2} \equiv \Gamma_{2 \mathrm{~L}}=\Gamma_{2 \mathrm{R}}=\bar{\Gamma}_{2 \mathrm{~L}}=\bar{\Gamma}_{2 \mathrm{R}}=$ $1,10,100,1000,10000$. As observed in Figs. 3 and 4, the difference $Q\left(\lambda_{1}, 0\right)-Q\left(\tilde{A}_{1}-\lambda_{1}, 0\right)$ is positive for $\lambda_{1}<\tilde{A}_{1} / 2$ and negative for $\lambda_{1}>\tilde{A}_{1} / 2$. Here, we only depict the difference for $\lambda_{1}<\tilde{A}_{1} / 2$. The other half has a similar structure if the absolute value of the difference is taken before the logarithm.

ratio of rate constants $\Gamma_{2} / \Gamma_{1}$ is increased by the same factor. Consequently, the single-current fluctuation theorem is well established in the large ratio limit $\Gamma_{2} / \Gamma_{1} \rightarrow \infty$. In this limit, the effective affinity is given by Eq. (80).

The effective affinity is depicted in Fig. 7 as a function of the energy $\beta \epsilon_{1}$ of the QD No. 1 for $\beta U=15$. We observe that the effective affinity takes the actual value (81) determined by the reservoirs for either low or large values of the energy $\beta \epsilon_{1}$. However, the effective affinity undergoes a significant reduction in between, down to a minimum of about $\tilde{A}_{1} \simeq$ $0.45 \times A_{1}$. The function has a characteristic shape, which can be explained in terms of the Fermi-Dirac distributions (15) and (16) entering in the expression (80) of the effective affinity. Away from their critical energy $\epsilon_{j}=\mu_{j}$ or $\epsilon_{j}=\mu_{j}-U$, these Fermi-Dirac distributions behave approximately as either constant functions or Maxwell-Boltzmann exponential distributions. Because of the logarithm defining the effective affinity (80), this latter switches between either constant or linear dependences on the energies or chemical potentials. Supposing that $\mu_{2 \mathrm{R}}<\epsilon_{2}<\mu_{2 \mathrm{~L}}-U$ and $0<U<\mu_{1 \mathrm{~L}}-\mu_{1 \mathrm{R}}$, we find that the effective affinity is given approximately by

$$
\tilde{A}_{1} \simeq \begin{cases}\beta\left(\mu_{1 \mathrm{~L}}-\mu_{1 \mathrm{R}}\right) & \text { for } \epsilon_{1}<\mu_{1 \mathrm{R}}-U \\ \beta\left(-\epsilon_{1}-U+\mu_{1 \mathrm{~L}}\right) & \text { for } \mu_{1 \mathrm{R}}-U<\epsilon_{1}<\mu_{1 \mathrm{R}} \\ \beta\left(\mu_{1 \mathrm{~L}}-\mu_{1 \mathrm{R}}-U\right) & \text { for } \mu_{1 \mathrm{R}}<\epsilon_{1}<\mu_{1 \mathrm{~L}}-U \\ \beta\left(\epsilon_{1}-\mu_{1 \mathrm{R}}\right) & \text { for } \mu_{1 \mathrm{~L}}-U<\epsilon_{1}<\mu_{1 \mathrm{~L}} \\ \beta\left(\mu_{1 \mathrm{~L}}-\mu_{1 \mathrm{R}}\right) & \text { for } \mu_{1 \mathrm{~L}}<\epsilon_{1}\end{cases}
$$

up to corrections that are smaller than $\beta=\left(k_{B} T\right)^{-1}$ in the zerotemperature limit $T \rightarrow 0$. Crossovers happen where the energy $\epsilon_{1}$ coincides with the values of the chemical potentials of the left- and right-hand reservoirs and the chemical potentials reduced by the Coulomb repulsion $U$. The slope of the effective 


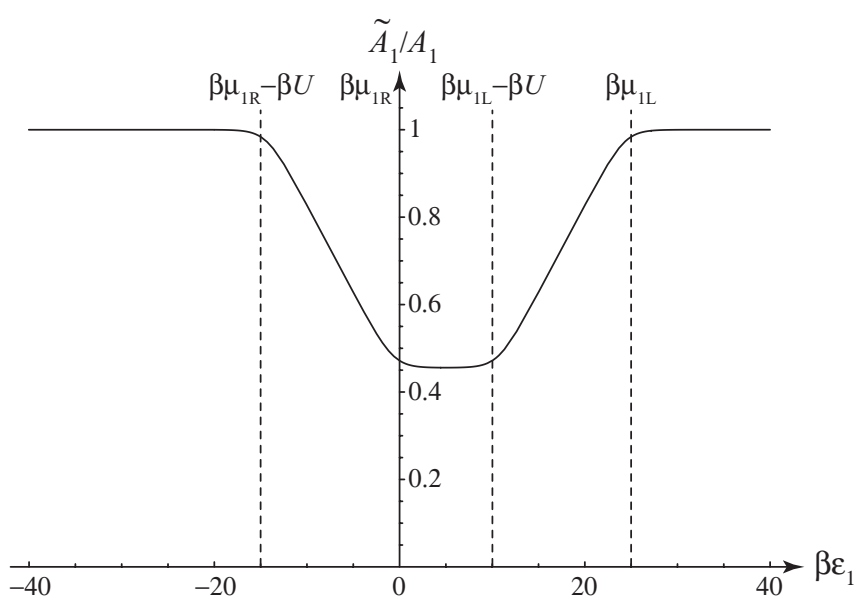

FIG. 7. The effective affinity (80) of the QD vs the dimensionless energy $\beta \epsilon_{1}$ of its level for the parameter values $\beta U=15$ and (84)-(90).

affinity versus $\beta \epsilon_{1}$ is successively $\{0,-1,0,+1,0\}$, as seen in Fig. 7. According to Eq. (91), the minimum value of the effective affinity is approximately given by $\tilde{A}_{1} \simeq A_{1}-\beta U=$ 10 in the middle interval $\beta \mu_{1 \mathrm{R}}=0<\beta \epsilon_{1}<\beta \mu_{1 \mathrm{~L}}-\beta U=$ 10. The affinity $A_{1}=25$ of the reservoirs is recovered for $\beta \epsilon_{1}<\beta \mu_{1 \mathrm{R}}-\beta U=-15$ and for $\beta \epsilon_{1}>\beta \mu_{1 \mathrm{~L}}=25$, which explains the features observed in Fig. 7 .

Equation (91) predicts that the minimum value of the effective affinity could be decreased further by increasing the Coulomb repulsion $U$. This is indeed the case, as is observed in Fig. 8, which depicts the effective affinity versus the energy $\epsilon_{1}$ now for the value (83) of the parameter $U$. Here, we see that the effective affinity may vary from the maximum value given by the affinity $A_{1}=25$ imposed by the reservoirs down to the very small minimum value $\widetilde{A}_{1} \simeq 0.083565$ at $\beta \epsilon_{1} \simeq-3.9525$, i.e., a drop by a factor 300 .

If the condition $\mu_{2 \mathrm{R}}<\epsilon_{2}<\mu_{2 \mathrm{~L}}-U$ is still satisfied for the parameter values of Fig. 8, the Coulomb repulsion is now larger than the difference of chemical potentials: $U>\mu_{1 \mathrm{~L}}-\mu_{1 \mathrm{R}}$. In

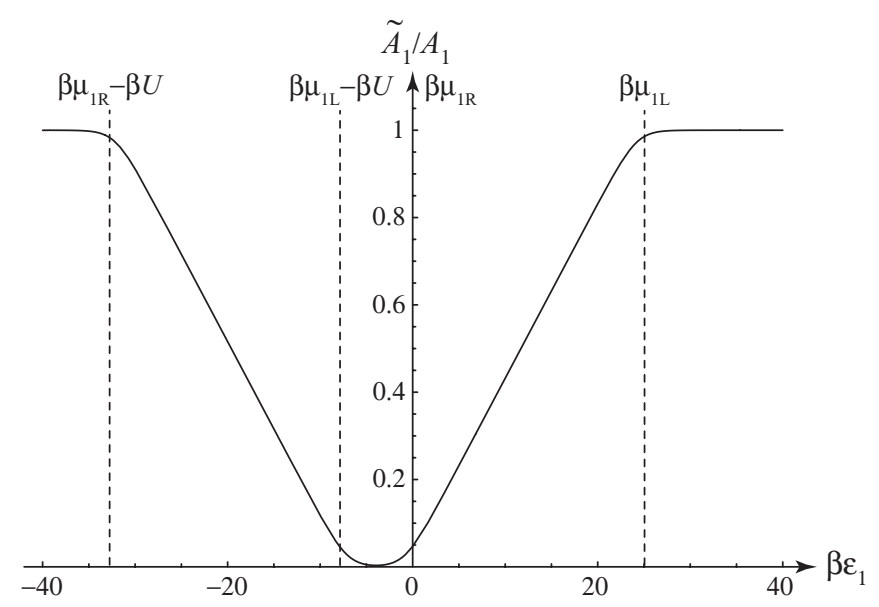

FIG. 8. The effective affinity (80) of the QD vs the dimensionless energy $\beta \epsilon_{1}$ of its level for the parameter values (83)-(90).

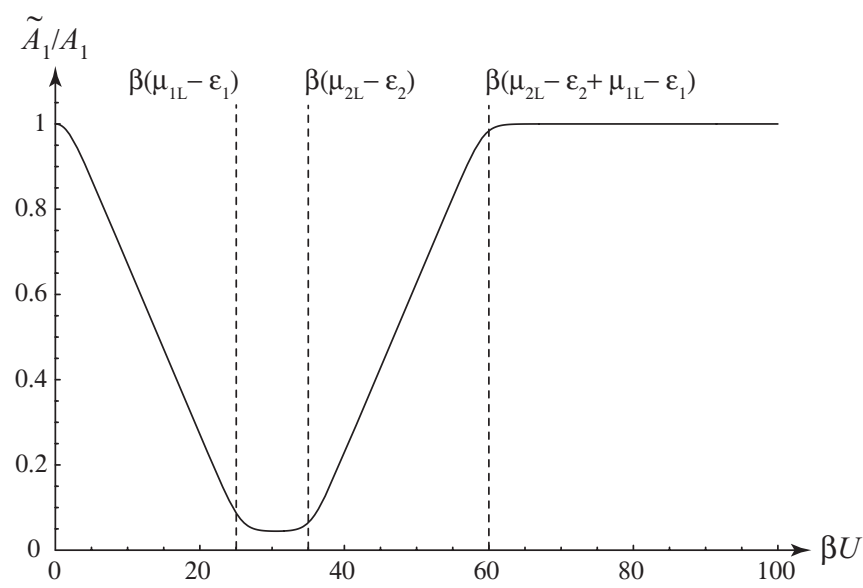

FIG. 9. The effective affinity (80) of the QD vs the dimensionless electrostatic coupling constant $\beta U$ for the parameter values $\beta \epsilon_{1}=0$ and (84)-(90).

this other regime, the effective affinity is given approximately by

$\tilde{A}_{1} \simeq \begin{cases}\beta\left(\mu_{1 \mathrm{~L}}-\mu_{1 \mathrm{R}}\right) & \text { for } \epsilon_{1}<\mu_{1 \mathrm{R}}-U \\ \beta\left(-\epsilon_{1}-U+\mu_{1 \mathrm{~L}}\right) & \text { for } \mu_{1 \mathrm{R}}-U<\epsilon_{1}<\mu_{1 \mathrm{~L}}-U \\ 0 & \text { for } \mu_{1 \mathrm{~L}}-U<\epsilon_{1}<\mu_{1 \mathrm{R}} \\ \beta\left(\epsilon_{1}-\mu_{1 \mathrm{R}}\right) & \text { for } \mu_{1 \mathrm{R}}<\epsilon_{1}<\mu_{1 \mathrm{~L}} \\ \beta\left(\mu_{1 \mathrm{~L}}-\mu_{1 \mathrm{R}}\right) & \text { for } \mu_{1 \mathrm{~L}}<\epsilon_{1}\end{cases}$

up to corrections that are smaller than $\beta=\left(k_{B} T\right)^{-1}$ in the zero-temperature limit $T \rightarrow 0$. In the middle interval $\beta \mu_{1 \mathrm{~L}}-$ $\beta U=-7.8<\beta \epsilon_{1}<\beta \mu_{1 \mathrm{R}}=0$, the minimum effective affinity reaches a value that vanishes in the low-temperature limit $T \rightarrow 0$. The actual value of the affinity $A_{1}=25$ is recovered for $\beta \epsilon_{1}<\beta \mu_{1 \mathrm{R}}-\beta U=-32.8$ or $\beta \epsilon_{1}>\beta \mu_{1 \mathrm{~L}}=25$.

The dependence of the effective affinity (80) on the Coulomb repulsion is shown in Fig. 9 for a given value of the energy $\beta \epsilon_{1}=0$. Here also, the effective affinity can be reduced down to a much lower value than the one determined by the reservoirs. By a similar reasoning to the one used to get Eqs. (91) and (92), we can obtain the approximate dependence of the effective affinity on the parameter $U$ under the conditions $\mu_{1 \mathrm{R}}-\epsilon_{1}<0<\mu_{1 \mathrm{~L}}-\epsilon_{1}<\mu_{2 \mathrm{~L}}-\epsilon_{2}$ as follows:

$$
\tilde{A}_{1} \simeq\left\{\begin{array}{l}
\beta\left(-U+\mu_{1 \mathrm{~L}}-\mu_{1 \mathrm{R}}\right) \\
\quad \text { for } 0<U<\mu_{1 \mathrm{~L}}-\epsilon_{1} \\
\beta\left(\epsilon_{1}-\mu_{1 \mathrm{R}}\right) \\
\quad \text { for } \mu_{1 \mathrm{~L}}-\epsilon_{1}<U<\mu_{2 \mathrm{~L}}-\epsilon_{2} \\
\beta\left(U+\epsilon_{2}-\mu_{2 \mathrm{~L}}+\epsilon_{1}-\mu_{1 \mathrm{R}}\right) \\
\quad \text { for } \mu_{2 \mathrm{~L}}-\epsilon_{2}<U<\mu_{2 \mathrm{~L}}-\epsilon_{2}+\mu_{1 \mathrm{~L}}-\epsilon_{1} \\
\beta\left(\mu_{1 \mathrm{~L}}-\mu_{1 \mathrm{R}}\right) \\
\quad \text { for } \mu_{2 \mathrm{~L}}-\epsilon_{2}+\mu_{1 \mathrm{~L}}-\epsilon_{1}<U
\end{array}\right.
$$

up to corrections smaller than $\beta=\left(k_{B} T\right)^{-1}$ as $T \rightarrow 0$. The piecewise linear approximation obtained from the Fermi-Dirac distributions here also explains the successive slopes -1 , 


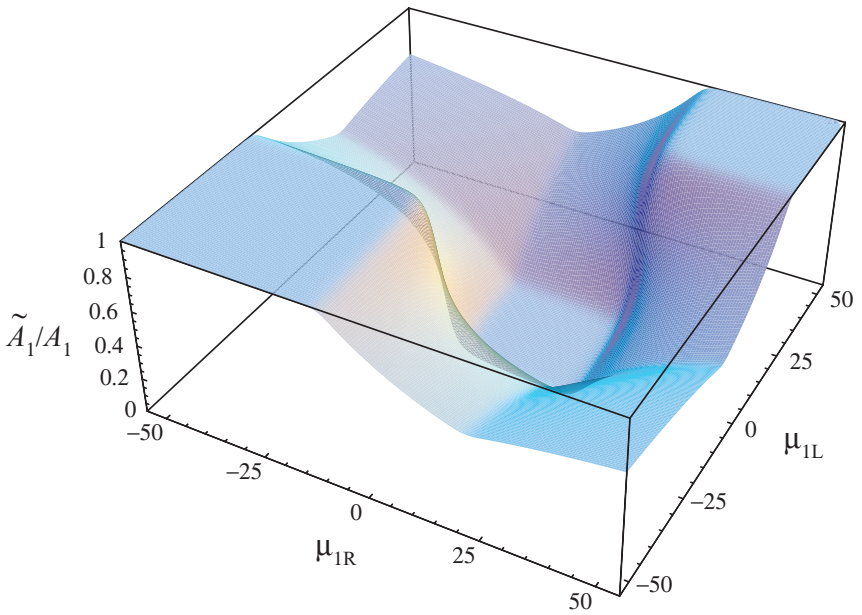

FIG. 10. (Color online) The effective affinity (80) divided by the actual affinity $A_{1}=\beta\left(\mu_{1 \mathrm{~L}}-\mu_{1 \mathrm{R}}\right)$ of the QD No. 1 vs the chemical potentials $\mu_{1 \mathrm{R}}$ and $\mu_{1 \mathrm{~L}}$ for the parameter values $\epsilon_{1}=-10, \epsilon_{2}=35$, $U=32.8, \Gamma_{1 \mathrm{~L}}=\Gamma_{1 \mathrm{R}}=\bar{\Gamma}_{1 \mathrm{~L}}=\bar{\Gamma}_{1 \mathrm{R}}=1, \Gamma_{2 \mathrm{~L}}=\Gamma_{2 \mathrm{R}}=\bar{\Gamma}_{2 \mathrm{~L}}=\bar{\Gamma}_{2 \mathrm{R}}=$ $10^{8}, \mu_{2 \mathrm{~L}}=70, \mu_{2 \mathrm{~L}}=0$, and the inverse temperature $\beta=1$. We notice that the two affinities $A_{1}$ and $\tilde{A}_{1}$ change their sign along the diagonal line $\mu_{1 \mathrm{~L}}=\mu_{1 \mathrm{R}}$ although their ratio does not.

$0,+1$, and 0 , observed in the plot of the effective affinity versus $\beta U$. We notice that the different linear pieces of the approximation match together at the crossover values of the variable $\beta U$. The minimum value is reached in the interval $\beta\left(\mu_{1 \mathrm{~L}}-\epsilon_{1}\right)=25<\beta U<\beta\left(\mu_{2 \mathrm{~L}}-\epsilon_{2}\right)=35$ while the affinity $A_{1}=25$ of the reservoirs is recovered for $\beta U>$ $\beta\left(\mu_{2 \mathrm{~L}}-\epsilon_{2}+\mu_{1 \mathrm{~L}}-\epsilon_{1}\right)=60$, as is indeed confirmed by Fig. 9.

Figure 10 shows how the effective affinity (80) behaves as a function of the chemical potentials $\mu_{1 \mathrm{R}}$ and $\mu_{1 \mathrm{~L}}$ of the reservoirs connected to the QD No. 1. This figure confirms that the effective affinity undergoes crossovers if these chemical potentials take the values $\epsilon_{1}$ and $\epsilon_{1}+U$. On the one hand, the effective affinity reaches its lower values in the domain where $\epsilon_{1}<\mu_{1 \mathrm{~L}}<\epsilon_{1}+U$ and $\epsilon_{1}<\mu_{1 \mathrm{R}}<\epsilon_{1}+U$. On the other hand, the actual value of the affinity is recovered in the domains $\mu_{1 \mathrm{~L}}, \mu_{1 \mathrm{~L}}<\epsilon_{1}$ and $\epsilon_{1}+U<\mu_{1 \mathrm{~L}}, \mu_{1 \mathrm{~L}}$. If the temperature increases, the effective affinity becomes smoother as we observe in Fig. 11 for a temperature five times higher.

In summary, the lowering of the effective affinity under specific conditions can be explained in the present model as the effect of the backaction of the secondary circuit interacting with the observed quantum dot. The charging and discharging rates of the quantum dot can be modified drastically by the coupling to the secondary circuit. In this way, the effective affinity can be much reduced in some regimes that are determined by the value of the energy $\epsilon_{1}$ of the quantum dot with respect to the values of the chemical potentials and the electrostatic coupling parameter $U$. This backaction effect tends to disappear as the temperature increases at constant voltages.

\section{CONCLUSIONS}

In the present paper, we have reported the study of the single-current fluctuation theorem in a Hamiltonian model

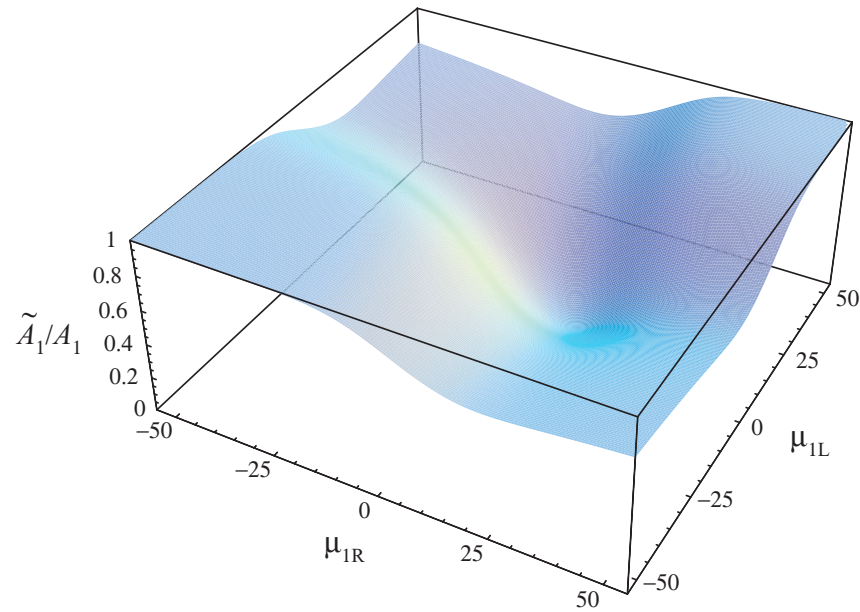

FIG. 11. (Color online) The effective affinity (80) divided by the actual affinity $A_{1}=\beta\left(\mu_{1 \mathrm{~L}}-\mu_{1 \mathrm{R}}\right)$ of the QD No. 1 vs the chemical potentials $\mu_{1 \mathrm{R}}$ and $\mu_{1 \mathrm{~L}}$ for the same parameter values as in Fig. 10 but the inverse temperature $\beta=0.2$. Here also, the two affinities $A_{1}$ and $\tilde{A}_{1}$ change their sign along the diagonal line $\mu_{1 \mathrm{~L}}=\mu_{1 \mathrm{R}}$ but their ratio does not.

of quantum electron transport in two capacitively coupled channels, each containing a quantum dot (QD). ${ }^{25}$ This system has similarities to the electronic devices used in typical counting statistics experiments ${ }^{20,37}$ where the current in one circuit can continuously monitor the state of the QD in the other circuit thanks to the capacitive coupling. The model allows us to investigate the effects of the backaction of the monitoring circuit on the counting statistics in light of the so-called fluctuation theorems.

Since both circuits are capacitively coupled and microreversibility holds for the total Hamiltonian (4), a fluctuation theorem is satisfied for the two currents flowing across the system. This two-current fluctuation theorem (39) or (41) relates the counting statistics of opposite random electron transfers in both circuits to the affinities or thermodynamic forces (35) and (36) driving the system away from equilibrium. The fluctuation theorem is valid far from equilibrium in the strongly nonlinear regimes encountered in electronic circuits composed of quantum dots.

However, in counting statistics experiments, one circuit is used to monitor the current fluctuations in the other circuit so that the counting statistics cannot be carried out on both currents together and is thus restricted to a single current. Accordingly, such experiments can only test a single-current fluctuation theorem. In general, the two-current fluctuation theorem does not imply the single-current fluctuation theorem except under certain conditions ${ }^{35}$ or in some limits, as we have demonstrated in the present paper.

In Sec. IV, we have studied the limit of large capacitive coupling between both circuits. In this limit, the state of simultaneous occupancy of both QD's in the two parallel channels is at such a high energy that it is energetically forbidden. The consequence is that the two single-occupancy states are separately accessible only from the empty state, and the single-current fluctuation theorem holds with respect to the affinity determined by the chemical potentials of the reservoirs. 
In Sec. $\mathrm{V}$, we have instead considered the limit where the current in one circuit is much larger than in the other circuit. Indeed, a large current ratio is a key feature of typical counting statistics experiments ${ }^{20,37}$ where the current ratio reaches values as high as $10^{7}-10^{8}$. The circuit with the very large current performs the continuous-time monitoring of the quantum state of the QD in the other circuit. In such a limit, the charging and discharging rates of the slow QD take values averaged over the very fast fluctuations of the monitoring circuit. This is the essence of the backaction of the monitoring circuit onto the QD circuit. As a consequence of the large current ratio limit, the single-current fluctuation theorem holds but with respect to the effective affinity (80), which can be significantly reduced with respect to the actual value of the affinity determined by the reservoirs of the corresponding circuit.

As shown in Sec. VI, e.g., by Eq. (91), the reduction of the affinity is due to the capacitive coupling between both circuits and occurs when the transition energies $\left\{\epsilon_{1}, \epsilon_{1}+U\right\}$ of the quantum dot lie within the bias window $\left[\mu_{1 \mathrm{R}}, \mu_{1 \mathrm{~L}}\right]$ where the dynamics of the system is sensitive to the fluctuations of the detector. In terms of the parameter $U$ of the Coulomb electrostatic interaction appearing in the Hamiltonian (1), the affinity is lowered according to $\tilde{A}_{1} \simeq A_{1}-\beta U$ under the validity conditions of Eq. (91). This result explicitly expresses the effect of the backaction between both circuits on the single-current fluctuation theorem. This backaction effect can be weakened if the Coulomb repulsion $U$ is decreased, but the monitoring circuit can no longer resolve the two states of the QD as in Fig. 2 if $U$ is too small. On the other hand, the backaction effect is also weakened for large values of the Coulomb repulsion as shown by Eq. (93) and in Fig. 9. Indeed, for a large Coulomb repulsion, the affinity recovers the value determined by the reservoirs and the backaction effect disappears. This case corresponds to the situation considered in Ref. 40, where a quantum fluctuation theorem was obtained in a multiple measurements scheme.

From a general viewpoint, the two-current fluctuation theorem implies the non-negativity of the entropy production in agreement with the second law of thermodynamics. The dissipation of energy can thus be evaluated in the electron transport process used to perform quantum measurement in the experiments of Refs. 20 and 37. This dissipation of energy accompanying quantum measurement is expected on fundamental grounds. ${ }^{41}$ The necessity of resolving the QD state in real time has as a direct consequence that the dissipation in the monitoring circuit is much higher than in the QD by a factor $\Pi_{2} / \Pi_{1}=\left(A_{2} / A_{1}\right) \times\left(J_{2} / J_{1}\right)$ of the same order of magnitude as the current ratio $J_{2} / J_{1}$. If the QD state is monitored with a sampling time $\Delta t$, the secondary circuit playing the role of the detector should have transitions on equal or shorter time scales according to Eq. (60). Since the secondary circuit is driven out of equilibrium by the affinity $A_{2}$, its electron current should satisfy $J_{2} \gtrsim(\Delta t)^{-1}$, so that the dissipated power should be bounded by $\Pi_{2}=k_{B} T A_{2} J_{2} \gtrsim k_{B} T A_{2}(\Delta t)^{-1}$. The higher the time resolution, the higher the dissipation rate.

To conclude, we have shown here that the single-current fluctuation theorem is valid under different limiting conditions and provides a fundamental understanding of the backaction effect of the monitoring circuit on the affinity of the monitored circuit, as observed in Ref. 20. The present study extends the analysis of Refs. 21, 23, and 24 in showing how the effective affinity of the single-current fluctuation theorem can be directly expressed in terms of the parameters entering the Hamiltonian of the system. The present study leaves open several issues. Although our study has been carried out with the simplified model of Ref. 25, which neglects the capacitances between the QD's and the reservoirs, we expect a similar reduction of the effective affinity if these capacitances are included in the model. Another issue is that the counting statistics experiments of Ref. 20 are performed with two QD's in series monitored by a quantum point contact, which form a system where the reduction of the affinity may be induced by more control parameters than the ones we have discussed here. Related issues concern experiments on fluctuation relations in quantum coherent conductors. ${ }^{42,43}$ We hope to report on these issues in forthcoming publications.

\section{ACKNOWLEDGMENTS}

The authors are grateful to David Andrieux for fruitful discussions as well as for communicating to them his results of July 2008 on the estimation of the effective affinity for the experiment reported in Ref. 20. G.B.C. thanks the "Fonds pour la Formation à la Recherche dans l'Industrie et l'Agriculture" (FRIA Belgium) for financial support. M.E. is supported by the Belgian Federal Government under the Interuniversity Attraction Pole project "NOSY" and by the European Union Seventh Framework Programme (FP7/2007-2013) under grant agreement 256251.

\section{APPENDIX: CALCULATION OF THE AVERAGE CURRENTS}

In this appendix, two different methods are developed in order to calculate the average currents given by Eq. (42) in terms of the leading eigenvalue $Q$ of the four-by-four matrix (31).

The first method starts from the eigenvalue equation (30) for the right eigenvector $\mathbf{v}$ associated with the eigenvalue $Q$ and from the adjoint equation

$$
\mathrm{L}^{\mathrm{T}} \cdot \mathbf{u}=-Q \mathbf{u}
$$

for the left eigenvector $\mathbf{u}$, where ${ }^{\mathrm{T}}$ denotes the transpose of the matrix. The left and right eigenvectors satisfy the normalization condition

$$
\mathbf{u}^{\mathrm{T}} \cdot \mathbf{v}=1 .
$$

Accordingly, the eigenvalue is given by

$$
Q=-\mathbf{u}^{\mathrm{T}} \cdot \mathrm{L} \cdot \mathbf{v} .
$$

Taking the partial derivative $\partial_{\alpha}$ with respect to the counting parameter $\lambda_{\alpha}$ of Eqs. (A2) and (A3) and using Eqs. (30) and (A1), we obtain the following expression for the average current:

$$
J_{\alpha}=\left.\partial_{\alpha} Q\right|_{\lambda=\mathbf{0}}=-\left.\mathbf{u}^{\mathrm{T}} \cdot \partial_{\alpha} \mathrm{L} \cdot \mathbf{v}\right|_{\lambda=\mathbf{0}} .
$$


Since the left and right eigenvectors are given at $\lambda=\mathbf{0}$ by

$$
\left.\mathbf{u}\right|_{\boldsymbol{\lambda}=\mathbf{0}}=\left(\begin{array}{l}
1 \\
1 \\
1 \\
1
\end{array}\right) \quad \text { and }\left.\quad \mathbf{v}\right|_{\lambda=\mathbf{0}}=\left(\begin{array}{c}
P_{00} \\
P_{10} \\
P_{01} \\
P_{11}
\end{array}\right)
$$

in terms of the probabilities (27), we get Eqs. (43) and (44) for the average currents.

With the second method, the average currents as well as the linear-response coefficients are directly calculated in terms of the characteristic determinant (37) of the four-by-four matrix (31). This determinant is a polynomial of fourth degree:

$$
Q^{4}+C_{3} Q^{3}+C_{2} Q^{2}+C_{1} Q+C_{0}=0,
$$

where the coefficients depend on the parameters of the model as well as on the counting parameters $\lambda_{1}$ and $\lambda_{2}$. We notice that the last coefficient is just the determinant of the matrix (31): $C_{0}=\operatorname{det} \mathrm{L}$.

Since the matrix (31) reduces to the matrix of a jump stochastic process conserving probability if $\lambda_{1}=\lambda_{2}=0$, the leading eigenvalue vanishes in this limit:

$$
Q(0,0)=0 \text {. }
$$

The average currents being given by Eq. (42), we take the partial derivative $\partial_{\alpha}$ of the characteristic determinant with respect to the counting parameter $\lambda_{\alpha}$ to get

$$
\begin{aligned}
& \left(4 Q^{3}+3 C_{3} Q^{2}+2 C_{2} Q+C_{1}\right) \partial_{\alpha} Q \\
& \quad+\partial_{\alpha} C_{3} Q^{3}+\partial_{\alpha} C_{2} Q^{2}+\partial_{\alpha} C_{1} Q+\partial_{\alpha} C_{0}=0 .
\end{aligned}
$$

Now, the counting parameters must be set equal to zero and, according to Eq. (A7), the average current is thus given by

$$
J_{\alpha}=-\left.\frac{\partial_{\alpha} C_{0}}{C_{1}}\right|_{\lambda=\mathbf{0}} .
$$

Using the symbolic manipulation software MATHEMATICA, ${ }^{44}$ we can evaluate the derivative $\partial_{\alpha} C_{0}$ if the reservoirs of the circuit $\alpha$ are at equilibrium, i.e., if its chemical potentials are equal so that its affinity is vanishing: $A_{\alpha}=\beta\left(\mu_{\alpha \mathrm{L}}-\mu_{\alpha \mathrm{R}}\right)=0$. The result is that this quantity vanishes under the condition $\Gamma_{j}=\bar{\Gamma}_{j}$ even if the other circuit is out of equilibrium, which establishes Eq. (48).

We notice that the Onsager coefficient can also be obtained in the same way. Using Eq. (46) and taking a further derivative of Eq. (A8) with respect to the other counting parameter $\lambda_{\beta}$, we get

$$
L_{\alpha, \beta}=L_{\beta, \alpha}=\left.\frac{\partial_{\alpha} \partial_{\beta} C_{0}}{2 C_{1}}\right|_{\lambda=\mathbf{0}, \mathbf{A}=\mathbf{0}},
$$

which is used to obtain Eq. (47) with the symbolic manipulation software MATHEMATICA. ${ }^{44}$
${ }^{1}$ D. J. Evans, E. G. D. Cohen, and G. P. Morriss, Phys. Rev. Lett. 71, 2401 (1993).

${ }^{2}$ G. Gallavotti and E. G. D. Cohen, Phys. Rev. Lett. 74, 2694 (1995).

${ }^{3}$ J. Kurchan, J. Phys. A 31, 3719 (1998).

${ }^{4}$ J. L. Lebowitz and H. Spohn, J. Stat. Phys. 95, 333 (1999).

${ }^{5}$ D. Andrieux and P. Gaspard, J. Stat. Mech. (2006) P01011.

${ }^{6}$ D. Andrieux and P. Gaspard, J. Stat. Phys. 127, 107 (2007).

${ }^{7}$ D. Andrieux, Nonequilibrium Statistical Thermodynamics at the Nanoscale (VDM Verlag, Saarbrücken, 2009).

${ }^{8}$ M. Esposito and C. Van den Broeck, Phys. Rev. Lett. 104, 090601 (2010).

${ }^{9}$ J. Tobiska and Yu. V. Nazarov, Phys. Rev. B 72, 235328 (2005).

${ }^{10}$ M. Esposito, U. Harbola, and S. Mukamel, Phys. Rev. B 75, 155316 (2007).

${ }^{11}$ U. Harbola, M. Esposito, and S. Mukamel, Phys. Rev. B 76, 085408 (2007).

${ }^{12}$ M. Esposito, U. Harbola, and S. Mukamel, Rev. Mod. Phys. 81, 1665 (2009).

${ }^{13}$ H. Förster and M. Büttiker, Phys. Rev. Lett. 101, 136805 (2008).

${ }^{14}$ K. Saito and Y. Utsumi, Phys. Rev. B 78, 115429 (2008).

${ }^{15}$ D. Andrieux, P. Gaspard, T. Monnai, and S. Tasaki, New J. Phys. 11, 043014 (2009).

${ }^{16}$ R. Sánchez, R. López, D. Sánchez, and M. Büttiker, Phys. Rev. Lett. 104, 076801 (2010).

${ }^{17}$ M. Campisi, P. Hänggi, and P. Talkner, Rev. Mod. Phys. 83, 771 (2011).

${ }^{18}$ T. De Donder and P. Van Rysselberghe, Affinity (Stanford University Press, Menlo Park, CA, 1936).
${ }^{19}$ H. B. Callen, Thermodynamics and an Introduction to Thermostatistics (Wiley, New York, 1985).

${ }^{20}$ T. Fujisawa, T. Hayashi, R. Tomita, and Y. Hirayama, Science 312, 1634 (2006).

${ }^{21}$ Y. Utsumi, D. S. Golubev, M. Marthaler, K. Saito, T. Fujisawa, and G. Schön, Phys. Rev. B 81, 125331 (2010).

${ }^{22} \mathrm{G}$.-L. Ingold and Yu. V. Nazarov, in Single Charge Tunneling, edited by H. Grabert and M. Devoret, NATO Advanced Studies Institute Series B: Physics, Vol. 294 (Plenum, New York, 1992), pp. 21-107.

${ }^{23}$ Y. Utsumi, D. S. Golubev, M. Marthaler, K. Saito, T. Fujisawa, and G. Schön, in Perspectives of Mesoscopic Physics: Dedicated to Yoseph Imry's 70th Birthday, edited by A. Aharony and O. EntinWohlman (World Scientific, Singapore, 2010), pp. 397-413.

${ }^{24}$ D. S. Golubev, Y. Utsumi, M. Marthaler, and G. Schön, Phys. Rev. B 84, 075323 (2011).

${ }^{25}$ G. Schaller, G. Kiesslich, and T. Brandes, Phys. Rev. B 82, 041303 (2010).

${ }^{26}$ D. T. McClure, L. DiCarlo, Y. Zhang, H.-A. Engel, C. M. Marcus, M. P. Hanson, and A. C. Gossard, Phys. Rev. Lett. 98, 056801 (2007).

${ }^{27}$ Y. Zhang, L. DiCarlo, D. T. McClure, M. Yamamoto, S. Tarucha, C. M. Marcus, M. P. Hanson, and A. C. Gossard, Phys. Rev. Lett. 99, 036603 (2007).

${ }^{28}$ G. Michalek and B. R. Bulka, Phys. Rev. B 80, 035320 (2009).

${ }^{29}$ C. Cohen-Tannoudji, J. Dupont-Roc, and G. Grynberg, Processus d'Interaction Entre Photons et Atomes (CNRS Editions, Paris, 1996).

${ }^{30}$ G. Schaller and T. Brandes, Phys. Rev. A 78, 022106 (2008). 
${ }^{31}$ U. Harbola, M. Esposito, and S. Mukamel, Phys. Rev. B 74, 235309 (2006).

${ }^{32}$ C. W. J. Beenakker, Phys. Rev. B 44, 1646 (1991).

${ }^{33}$ N. G. van Kampen, Stochastic Processes in Physics and Chemistry (North-Holland, Amsterdam, 1981).

${ }^{34}$ J. Schnakenberg, Rev. Mod. Phys. 48, 571 (1976).

${ }^{35}$ D. Andrieux and P. Gaspard, C. R. Phys. 8, 579 (2007).

${ }^{36}$ D. Andrieux and P. Gaspard, J. Stat. Mech. (2007) P02006.

${ }^{37}$ S. Gustavsson, R. Leturcq, B. Simovič, R. Schleser, T. Ihn, P. Studerus, K. Ensslin, D. C. Driscoll, and A. C. Gossard, Phys. Rev. Lett. 96, 076605 (2006).

${ }^{38}$ D. T. Gillespie, J. Comput. Phys. 22, 403 (1976).

${ }^{39}$ D. T. Gillespie, J. Phys. Chem. 81, 2340 (1977).
${ }^{40}$ M. Campisi, P. Talkner, and P. Hänggi, Phys. Rev. Lett. 105, 140601 (2010).

${ }^{41}$ J. von Neumann, Mathematical Foundations of Quantum Mechanics (Princeton University Press, Princeton, NJ, 1955).

${ }^{42}$ S. Nakamura, Y. Yamauchi, M. Hashisaka, K. Chida, K. Kobayashi, T. Ono, R. Leturcq, K. Ensslin, K. Saito, Y. Utsumi, and A. C. Gossard, Phys. Rev. Lett. 104, 080602 (2010).

${ }^{43}$ S. Nakamura, Y. Yamauchi, M. Hashisaka, K. Chida, K. Kobayashi, T. Ono, R. Leturcq, K. Ensslin, K. Saito, Y. Utsumi, and A. C. Gossard, Phys. Rev. B 83, 155431 (2011).

${ }^{44}$ S. Wolfram, Mathematica (Addison-Wesley, Redwood City, CA, 1988). 